\title{
Extended Weyl Invariance in a Bimetric Model and Partial Masslessness
}

\author{
S.F. Hassan, ${ }^{1}$ Angnis Schmidt-May, ${ }^{2}$ Mikael von Strauss ${ }^{3}$ \\ ${ }^{1}$ Department of Physics \& The Oskar Klein Centre, \\ Stockholm University, AlbaNova University Centre, SE-106 91 Stockholm, Sweden \\ ${ }^{2}$ Institut für Theoretische Physik, Eidgenössische Technische Hochschule Zürich \\ Wolfgang-Pauli-Strasse 27, 8093 Zürich, Switzerland \\ ${ }^{3}$ UPMC-CNRS, UMR7095, Institut d'Astrophysique de Paris, GReCO, \\ 98bis boulevard Arago, F-75014 Paris, France. \\ E-mail: fawad@fysik.su.se, angniss@itp.phys.ethz.ch, strauss@iap.fr
}

\begin{abstract}
We revisit a particular ghost-free bimetric model which is related to both partial masslessness (PM) and conformal gravity. Linearly, the model propagates six instead of seven degrees of freedom not only around de Sitter but also around flat spacetime. Nonlinearly, the equations of motion can be recast in the form of expansions in powers of curvatures, and exhibit a remarkable amount of structure. In this form, the equations are shown to be invariant under scalar gauge transformations, at least up to six orders in derivatives, the lowest order term being a Weyl scaling of the metrics. The terms at two-derivative order reproduce the usual PM gauge transformations on de Sitter backgrounds. At the fourderivative order, a potential obstruction that could destroy the symmetry is shown to vanish. This in turn guarantees the gauge invariance to at least six-orders in derivatives. This is equivalent to adding up to 10-derivative corrections to conformal gravity. More generally, we outline a procedure for constructing the gauge transformations order by order as an expansion in derivatives and comment on the validity and limitations of the procedure. We also discuss recent arguments against the existence of a PM gauge symmetry in bimetric theory and show that, at least in their present form, they are evaded by the model considered here. Finally, we argue that a bimetric approach to PM theory is more promising than one based on the existence of a fundamental PM field.
\end{abstract}

KEYWORDS: modified gravity, bimetric gravity, Weyl invariance, higher spin fields 


\section{Contents}

1 Motivation and summary of results $\quad 1$

2 Review of the derivative expansion in bimetric theory $\quad 4$

2.1 The bimetric action 4

2.2 Perturbative expansion of bimetric equations 5

2.3 A model with a possible gauge symmetry 7

3 Linear analysis: PM theory, conformal gravity, and the bimetric model $\quad 8$

3.1 Partial masslessness in the Fierz-Pauli framework 8

3.2 Linearised conformal gravity and PM in bimetric framework 9

$\begin{array}{lll}3.3 & \text { Linear theory in flat space } & 12\end{array}$

4 A systematic study of the extended Weyl symmetry 13

4.1 General form of symmetry transformations 13

4.2 Structure of the derivative expansion of the bimetric equations $\quad 15$

4.3 Perturbative bootstrap construction of gauge symmetry 16

4.4 Relation to the partially massless gauge symmetry 20

4.5 Limitations of the perturbative expansion 21

5 Discussion $\quad 22$

5.1 Summary and discussion of results 22

5.2 Discussion of the counter-arguments 24

A Details of bimetric theory and its curvature expansion $\quad 27$

A.1 Structure of action and equations of motion 27

A.2 Obtaining the curvature expansions 28

B Four-derivative terms in gauge transformations $\quad 29$

B.1 Higher-curvature equations 29

B.2 Vanishing of the four-derivative contributions 30

\section{Motivation and summary of results}

The two major unresolved issues in General Relativity (GR) are the non-renormalisability of the theory and the cosmological constant problem. One expects that a theory of spin-2 fields 
with more symmetry than GR will be better behaved in these respects. One such theory is conformal gravity [1] defined by the action,

$$
S_{\mathrm{CG}}=\int \mathrm{d}^{4} x \sqrt{g} W_{\mu \nu \rho \sigma} W^{\mu \nu \rho \sigma} \sim \int \mathrm{d}^{4} x \sqrt{g}\left(R_{\mu \nu} R^{\mu \nu}-\frac{1}{3} R^{2}\right)
$$

in terms of the Weyl (W) or Ricci (R) tensors; the two formulations differ only by the topological Euler density. In addition to diffeomorphism invariance this action is invariant under a Weyl scaling of the metric. This is a 4-derivative theory and propagates 6 modes, consisting of a massless spin-2 field and 4 ghost modes [2-7]. Despite having an extra symmetry as compared to GR, the presence of the ghost instability makes the theory less attractive. ${ }^{1}$

Another theory with a novel gauge symmetry is the linear partially massless (PM) theory of a massive spin-2 field in an Einstein-de Sitter (EdS) background [11-13]. Due to the symmetry, the spin-2 field propagates 4 (instead of the usual 5) polarisations. To this, one can add a linear massless graviton (regarding the PM field itself as the graviton requires giving up general covariance). The spectrum is now similar to that of conformal gravity linearised around EdS backgrounds [7]. However, while ghost-free, this theory is non-interacting and exists only around special (EdS) backgrounds. The question is if there exists a unitary theory of interacting spin-2 fields with a PM-like gauge symmetry. ${ }^{2}$ The first investigations involved explicit construction of cubic interaction vertices [14-16], but problems are encountered at the quartic level.

On the other hand, a nonlinear setup with the right general features is the ghost-free bimetric theory [17]. There exists a unique bimetric model that, when linearised around EdS backgrounds, contains a massless and a partially massless spin-2 field, exhibits linear PM gauge symmetry and also up to realises the global part of the PM transformations nonlinearly $[18,19]$. But this is far from establishing the full gauge invariance of the nonlinear model. It turns out that the model also exhibits features of conformal gravity. In a derivative expansion, it reproduces the conformal gravity equation of motion at the lowest order [20] and, hence, is invariant under local Weyl scalings to this order. In this paper we explore this property beyond the lowest order and discuss its relevance to the presence or absence of PM symmetry. We show that the Weyl scalings can be extended at least up to six-derivative terms to maintain the symmetry of the equations. When restricted to the linear theory in EdS backgrounds, one recovers the standard PM transformations. The bimetric model provides a unified description of linear PM theory and conformal gravity up to higher derivative corrections. More work is needed to find if this structure can be extended to all orders.

Another potential setup for PM symmetry is nonlinear massive gravity [21], which is ghost-free [22] and can also be formulated around any background, including EdS spacetimes [23-25]. A specific massive gravity model, with a fixed dS reference metric, was identified in

\footnotetext{
${ }^{1}$ Nonlinear infinite derivative generalisations geared towards avoiding the ghost problem have been considered in e.g. [8-10], but the Weyl symmetry is then generically destroyed.

${ }^{2}$ To be of relevance to the renormalisation and the cosmological constant problems, the PM symmetry must also affect the physical gravitational field.
} 
[26] as exhibiting PM symmetry in the decoupling limit. However, it was soon argued that this action lacks the gauge symmetry at the nonlinear level $[27-29] .^{3}$

There have also been arguments against PM symmetry in the nonlinear bimetric model. Since massive gravity models can be regarded as a limit of bimetric models around given solutions, it has been argued, for instance in [27, 29], that ruling out PM symmetry in massive gravity, implies the same for the bimetric model. ${ }^{4}$ However, [31] argued that if the bimetric model indeed had a PM symmetry, it would most likely be destroyed in the massive gravity limit. These results are supported by the findings in the present work. Other arguments are discussed in the last section. Recent work on the subject of partial masslessness includes $[32-35]$.

Summary of results: This paper does not directly deal with PM symmetry and the possibility of its realisation in a nonlinear theory. Rather, we explicitly investigate a particular bimetric model in a derivative expansion. On eliminating one of the metrics between the two equations, one obtains the 4-derivative conformal gravity equation of motion which is invariant under Weyl scaling of the metrics [20]. Here we investigate the possibility of extending the zero-derivative gauge symmetry to higher orders in derivatives. The structure of the equations enables us to find a prescription for constructing the gauge transformations order by order,

$$
\begin{aligned}
& \Delta g_{\mu \nu}=\Delta_{(0)} g_{\mu \nu}+\Delta_{(2)} g_{\mu \nu}+\Delta_{(4)} g_{\mu \nu}+\Delta_{(6)} g_{\mu \nu}+\ldots \\
& \Delta f_{\mu \nu}=\Delta_{(0)} f_{\mu \nu}+\Delta_{(2)} f_{\mu \nu}+\Delta_{(4)} f_{\mu \nu}+\Delta_{(6)} f_{\mu \nu}+\ldots
\end{aligned}
$$

which leave the equations of motion invariant on-shell up to sixth order in derivatives. Here, $\Delta_{(0)} g_{\mu \nu}=\phi g_{\mu \nu}$ and $\Delta_{(0)} f_{\mu \nu}=\phi f_{\mu \nu}$ are Weyl scalings of the metrics and each $\Delta_{(2 n)}$ contains terms with $2 n$ derivatives. The prescription insures that terms with $2 n=(4 m+2)$ derivatives (for integer $m$ ) in the transformation can always be constructed. But the existence of the $2 n=4 m$-derivative terms requires the model to satisfy certain conditions. It is explicitly demonstrated that these conditions are satisfied for the four-derivative term and thus we are able to show the invariance of the equations of motion up to six orders in derivatives. This is equivalent to considering up to 10-derivative corrections to the 4-derivative Bach equation.

On Einstein-de Sitter backgrounds, the 2-derivative terms in the transformations of the nonlinear metrics reduce to the well-known gauge transformations for a partially massless spin-2 perturbation. This suggests that (1.2) may be considered as potential extensions of the linear PM gauge symmetry. But wether such a gauge symmetry really exists, remains to be seen. In this setup, the metrics are not restricted to de Sitter backgrounds and hence we can also study the behaviour of the model around other backgrounds. Unlike its massive

\footnotetext{
${ }^{3}$ The analysis of constraints in [30] rules out massive gravity with 4 polarisations for a Minkowski reference metric which is consistent with the linear PM theory.

${ }^{4}$ The massive gravity limit of the bimetric model in [18] does not seem to reproduce the massive gravity PM candidate identified in [26], but is consistent with the parameters values found in the subsequent work $[28]$.
} 
gravity counterpart, the bimetric model also has flat background solutions which enable us to extend the notion of linear partial masslessness to Minkowski space. One finds that around flat backgrounds no decomposition into spin-2 mass eigenstates exists, instead, the spectrum coincides with the conformal gravity spectrum around flat spacetime, i.e., two massless tensors and one massless vector. The results are discussed in more detail in the text and in section 5 .

The paper is organised as follows. In section 2 we review details of ghost-free bimetric theory, the perturbative expansion of its equations of motion, and the emergence of the Bach equation in a particular bimetric model. Section 3 considers this model at the quadratic level and the relation to both linear PM theory and linear conformal gravity, including the flat background case. In section 4 we study the perturbative expansions of equations in this model and outline the procedure for constructing the higher-derivative terms in the gauge transformations of the metrics. We establish the invariance to sixth order in derivatives, and discuss the relation to PM transformations, as well as the limitations of the procedure. Our results are discussed in section 5, where we also comment on various counter arguments and no-go results in the recent literature. Some technical details are provided in the appendices.

\section{Review of the derivative expansion in bimetric theory}

Here we briefly review the structure of ghost-free bimetric theory and outline how to derive the higher-curvature expansions of its equations of motion. The main results are summarised in this sections; some more details can be found in appendix A.

\subsection{The bimetric action}

The ghost-free action for two spin-2 fields $g_{\mu \nu}$ and $f_{\mu \nu}$ with non-derivative interactions is [17],

$$
\mathcal{S}(g, f)=\int \mathrm{d}^{4} x\left[m_{g}^{2} \sqrt{g} R(g)+m_{f}^{2} \sqrt{f} R(f)-2 m^{4} \sqrt{g} V\left(\sqrt{g^{-1} f}\right)\right],
$$

where, $m_{g}$ and $m_{f}$ are the two Planck masses and $m$ is an additional mass scale. The potential is given in terms of a square-root matrix $S \equiv \sqrt{g^{-1} f}$ as,

$$
V(S)=\sum_{n=0}^{4} \frac{\beta_{n}}{n !(4-n) !} \epsilon_{\mu_{1} \cdots \mu_{n} \lambda_{n+1} \cdots \lambda_{4}} \epsilon^{\nu_{1} \cdots \nu_{n} \lambda_{n+1} \cdots \lambda_{4}} S_{\nu_{1}}^{\mu_{1}} \cdots S_{\nu_{n}}^{\mu_{n}} \equiv \sum_{n=0}^{4} \beta_{n} e_{n}(S) .
$$

where $\beta_{n}$ are five interaction parameters. The $e_{n}(S)$ are the elementary symmetric polynomials of the eigenvalues of $S$; for their definitions see appendix A.1. ${ }^{5}$

The fact that both $g_{\mu \nu}$ and $f_{\mu \nu}$ in (2.1) have Einstein-Hilbert kinetic terms is related to the invariance of the potential under the interchanges $g \leftrightarrow f$ and $\beta_{n} \leftrightarrow \beta_{4-n}$. This invariance directly follows from the identity [17],

$$
\sqrt{g} \sum_{n=0}^{4} \beta_{n} e_{n}(S)=\sqrt{f} \sum_{n=0}^{4} \beta_{4-n} e_{n}\left(S^{-1}\right) .
$$

\footnotetext{
${ }^{5}$ For a non-dynamical $f_{\mu \nu}=\eta_{\mu \nu}$ and a restricted set of $\beta_{n}, V(S)$ becomes the massive gravity potential first proposed in $[21,36]$ and proven to be free of the Boulware-Deser ghost at the nonlinear level in [22, 24, 25].
} 
A Hamiltonian analysis shows that this theory propagates 7 modes $[17,37]$ and no BoulwareDeser ghost $[38,39]$. Around backgrounds of the type $\bar{f}_{\mu \nu}=c^{2} \bar{g}_{\mu \nu}$, these modes combine into massless and massive spin-2 fluctuations with, respectively, 2 and 5 polarisations [40]. In general, a bimetric theory can be interpreted as describing a gravitational metric $g_{\mu \nu}$ (with standard matter couplings) in the presence of an extra spin-2 field $f_{\mu \nu}$. Then, for $m_{f}<<m_{g}=M_{p}$, the physical metric $g_{\mu \nu}$ is a mostly massless field (in contrast to the massive gravity limit $\left.m_{f} \rightarrow \infty\right)$ [40, 41].

\subsection{Perturbative expansion of bimetric equations}

The equations of motion obtained on varying the action (2.1) with respect to $g_{\mu \nu}$ and $f_{\mu \nu}$ are of the form,

$$
\begin{array}{ll}
g \text {-eom : } & \frac{1}{\mu^{2}}\left(R^{\mu}{ }_{\nu}-\frac{1}{2} \delta^{\mu}{ }_{\nu} R\right)+V_{\nu}^{\mu}(S)=0, \\
f \text {-eom : } & \frac{\alpha^{2}}{\mu^{2}}\left(\tilde{R}^{\mu}{ }_{\nu}-\frac{1}{2} \delta^{\mu}{ }_{\nu} \tilde{R}\right)+\tilde{V}_{\nu}^{\mu}\left(S^{-1}\right)=0 .
\end{array}
$$

In these expressions, $R_{\mu \nu}$ and $\tilde{R}_{\mu \nu}$ are the curvatures of $g_{\mu \nu}$ and $f_{\mu \nu}$, respectively, and $V_{\mu \nu}$ and $\tilde{V}_{\mu \nu}$ are the corresponding interaction contributions, explicitly given in appendix A.1. Moreover, $S=\sqrt{g^{-1} f}$. In the $g$-equation, the first index is raised by $g^{\mu \nu}$, and in the $f$ equation by $f^{\mu \nu}$. To simplify the expressions, we use the notation,

$$
\mu^{2} \equiv \frac{m^{4}}{m_{g}^{2}}, \quad \alpha \equiv \frac{m_{f}}{m_{g}},
$$

The $g$-equation depends on $f_{\mu \nu}$ (through $S$ ) but does not contain derivatives of $f_{\mu \nu}$. Hence, in principle, it can be algebraically solved for $f_{\mu \nu}$ in terms of $R_{\mu \nu}(g) / \mu^{2}$. For example, when $\beta_{0}$ and $\beta_{1}$ are the only non-vanishing parameters, the potential is $V=\beta_{0}+\beta_{1} \operatorname{Tr}(S)$ and its variation gives $V_{\nu}^{\mu}(S)=\beta_{0} \delta^{\mu}{ }_{\nu}+\beta_{1}\left(S^{\mu}{ }_{\nu}-\delta^{\mu}{ }_{\nu} \operatorname{Tr}(S)\right)$. In this case, (2.4a) yields the exact expression,

$$
S_{\nu}^{\mu}=-\frac{\beta_{0}}{3 \beta_{1}} \delta_{\nu}^{\mu}+\frac{1}{\beta_{1} \mu^{2}} g^{\mu \rho} P_{\rho \nu},
$$

where $P_{\mu \nu}$ is the (scaled) Schouten tensor of $g_{\mu \nu}$,

$$
P_{\mu \nu}=R_{\mu \nu}-\frac{1}{6} g_{\mu \nu} R \text {. }
$$

Since $f_{\mu \nu}=g_{\mu \rho}\left(S^{2}\right)^{\rho}{ }_{\nu}$, one immediately obtains,

$$
f_{\mu \nu}=\frac{\beta_{0}^{2}}{9 \beta_{1}^{2}} g_{\mu \nu}-\frac{2 \beta_{0}}{3 \beta_{1}^{2} \mu^{2}} P_{\mu \nu}+\frac{1}{\beta_{1}^{2} \mu^{4}} P_{\mu \rho} g^{\rho \sigma} P_{\sigma \nu} .
$$

This is simply a rewriting of the $g$-equation, but does not involve solving it as a differential equation. Using this in the $f$-equation $(2.4 \mathrm{~b})$, gives a higher derivative equation for $g_{\mu \nu}$ alone.

For generic $\beta_{n}$ parameters in (2.4a), it is not easy to obtain an exact solution for $S$, and hence for $f=g S^{2}$. But, for small $R_{\mu \nu} / \mu^{2}$, it is always possible to find a perturbative solution 
[20]. At lowest order, i.e., neglecting $R_{\mu \nu} / \mu^{2}$, this gives $f_{\mu \nu}=a^{2} g_{\mu \nu}+\cdots$, with the constant $a$ determined by the polynomial equation $\left.V_{\nu}^{\mu}\right|_{S=a 1}=0$. Curvature corrections to this can be systematically computed and one arrives at an expression for $f_{\mu \nu}$ of the form [20],

$$
f_{\mu \nu}=a^{2} g_{\mu \nu}+\frac{b}{\mu^{2}} P_{\mu \nu}+\frac{c_{1}}{\mu^{4}} P_{\mu \nu}^{2}+\frac{c_{2}}{\mu^{4}}\left[\frac{1}{3} e_{2}(P) g_{\mu \nu}-P P_{\mu \nu}\right]+\mathcal{O}\left(\frac{P^{3}}{\mu^{6}}\right)
$$

where indices on the right-hand side are contracted with $g_{\mu \nu}$. The coefficients $a, b$ and $c_{n}$ are given in terms of bimetric parameters in appendix A.2. Equation (2.9) is a rewriting of the $g$-equation (2.4a) and is satisfied by the compatible solutions of the latter perturbatively, ${ }^{6}$ as long as curvatures are small compared to the mass scale $\mu=m^{2} / m_{g}$. In principle, the coefficients in $(2.9)$ can be determined to arbitrary order, and terms with $2 n$ derivatives are suppressed by $\mu^{2 n}$.

Alternatively, we can obtain a solution for $g_{\mu \nu}$ from the $f$-equation $(2.4 \mathrm{~b})$. It has a very similar form,

$$
g_{\mu \nu}=\tilde{a}^{2} f_{\mu \nu}+\frac{\tilde{b}}{\tilde{\mu}^{2}} \tilde{P}_{\mu \nu}+\frac{\tilde{c}_{1}}{\tilde{\mu}^{4}} \tilde{P}_{\mu \nu}^{2}+\frac{\tilde{c}_{2}}{\tilde{\mu}^{4}}\left[\frac{1}{3} e_{2}(\tilde{P}) f_{\mu \nu}-\tilde{P} \tilde{P}_{\mu \nu}\right]+\mathcal{O}\left(\frac{\tilde{P}^{3}}{\tilde{\mu}^{6}}\right)
$$

where $\tilde{P}_{\mu \nu} \equiv P_{\mu \nu}(f)$ is the Schouten tensor for $f_{\mu \nu}$. Indices are contracted with $f_{\mu \nu}$ and the suppressing mass scale is now $\tilde{\mu}=\mu / \alpha$.

For generic models, the two expansions (2.9) and (2.10) are not simultaneously valid. For example, (2.9) at the lowest order reads $f_{\mu \nu}=a^{2} g_{\mu \nu}+\cdots$, whereas (2.10) is of the form $g_{\mu \nu}=\tilde{a}^{2} f_{\mu \nu}+\cdots$. Obviously, a necessary condition for the validity of both expansions is that $\tilde{a}^{2}=a^{2}$ (which can be satisfied by fixing one of the $\beta_{n}$ ). The model we consider in this paper satisfies this property.

It is possible to use the expression (2.9) for $f_{\mu \nu}$ to eliminate it from the $f$-equation (2.4b). This yields the following higher derivative equation for $g_{\mu \nu}$,

$$
\begin{aligned}
& x_{00} g_{\mu \nu}+\frac{x_{10}}{\mu^{2}} \mathcal{G}_{\mu \nu}+\frac{x_{11}}{\mu^{2}} P_{\mu \nu}+\frac{x_{20}}{\mu^{4}} B_{\mu \nu} \\
& +\frac{x_{21}}{\mu^{4}}\left[\left(s_{1}+2 s_{2}\right) P_{\mu}{ }^{\rho} P_{\rho \nu}-2 s_{2} P P_{\mu \nu}-\frac{s_{2}}{3} g_{\mu \nu}\left(P_{\rho \sigma} P^{\rho \sigma}-P^{2}\right)\right] \\
& -\frac{x_{22}}{\mu^{4}}\left[3 P P_{\mu \nu}-2 P_{\mu}{ }^{\rho} P_{\rho \nu}-\frac{1}{2} g_{\mu \nu}\left(P^{2}-P^{\alpha \beta} P_{\alpha \beta}\right)\right]+\mathcal{O}\left(\frac{P^{3}}{\mu^{6}}\right)=0 .
\end{aligned}
$$

The coefficients $x_{m n}$ and $s_{n}$ are given in appendix A.2, and $\mathcal{G}_{\mu \nu}=R_{\mu \nu}-\frac{1}{2} g_{\mu \nu} R$ is the Einstein tensor of $g_{\mu \nu}$. We have collected some of the four-derivative terms into the Bach tensor [1],

$$
B_{\mu \nu}=-\nabla^{2} P_{\mu \nu}-\nabla_{\mu} \nabla_{\nu} P_{\rho}^{\rho}+\nabla_{\rho} \nabla_{\mu} P_{\nu}^{\rho}+\nabla_{\rho} \nabla_{\nu} P_{\mu}^{\rho}-2 P_{\mu}{ }^{\rho} P_{\rho \nu}+\frac{1}{2} g_{\mu \nu} P^{\rho \sigma} P_{\rho \sigma} .
$$

\footnotetext{
${ }^{6}$ Let us comment on the generality of such expansions. If $g_{\mu \nu}$ is an Einstein metric, then $P_{\mu \nu}=(\Lambda / 3) g_{\mu \nu}$ and (2.9) implies $f_{\mu \nu}=c^{2} g_{\mu \nu}$, for some constant $c^{2}$. On the other hand, in generic bimetric models when one metric is Einstein, the other one is Einstein too, but the two metrics are not necessarily proportional to each other. Obviously, such non-proportional Einstein solutions are not captured by (2.9). However, in classes of bimetric models, the $\beta_{i}$-models (where only one $\beta_{i}$ out of $\beta_{1}, \beta_{2}, \beta_{3}$ is non-zero), when either metric is Einstein, one necessarily has $f_{\mu \nu}=c^{2} g_{\mu \nu}$, as implied by (2.9) [31]. In this paper we will work with a $\beta_{2}$-model.
} 
Note that the Bach equation, $B_{\mu \nu}=0$, is the equation of motion for conformal gravity.

Equation (2.11) can be rewritten as an Einstein equation for $g_{\mu \nu}$ with higher derivative corrections. The highest number of derivatives on the same $g_{\mu \nu}$ is four; these appear in $B_{\mu \nu}$ and in some higher order terms which all arise from expanding the Einstein tensor $\tilde{\mathcal{G}}_{\mu \nu}$ of $f_{\mu \nu}$. All other terms, including the corrections, contain a maximum of two derivatives on $g_{\mu \nu}$, but in higher powers. This is consistent with the fact that we need to specify four initial conditions in the original bimetric equations.

To summarise, we have re-expressed equations (2.4a) and (2.4b) as (2.9) and (2.11). The perturbative equivalence between these two sets of equations holds algebraically and the solutions of the earlier set satisfy (2.11) perturbatively (subject to the comment in footnote 6). For the purpose of this paper, it is not necessary that these two sets are also equivalent as differential equations. In particular, studying the symmetry properties of the equations involves only algebraic manipulations and, for such purposes, the two sets of equations can be treated perturbatively equivalent.

\subsection{A model with a possible gauge symmetry}

Let us consider a particular bimetric model that leads to an equation (2.11) for $g_{\mu \nu}$ with,

$$
x_{00}=x_{10}=x_{11}=0,
$$

so that the equation starts at fourth order in derivatives. Using the expressions for the $x_{m n}$, it can easily be shown that these conditions uniquely fix the bimetric interaction parameters to the following values,

$$
\beta_{1}=\beta_{3}=0, \quad \alpha^{4} \beta_{0}=3 \alpha^{2} \beta_{2}=\beta_{4} .
$$

It turns out that this choice of parameters also sets $x_{21}=x_{22}=0$ and thus specifies a bimetric theory whose equations of motion imply [20],

$$
B_{\mu \nu}+\mathcal{O}\left(\frac{P^{3}}{\mu^{6}}\right)=0 .
$$

At the lowest order, this is the equation of motion for conformal gravity, $B_{\mu \nu}=0$, which is invariant under Weyl scalings $g_{\mu \nu} \rightarrow e^{\phi(x)} g_{\mu \nu}$, since $B_{\mu \nu} \rightarrow e^{-\phi(x)} B_{\mu \nu}$. Hence, at the lowest order in a curvature expansion, the equations of the bimetric model (2.14) share the Weyl symmetry of conformal gravity. The corresponding transformation of $f_{\mu \nu}$ can be obtained from (2.9). The question is if this is just an accidental symmetry of the bimetric equations at the four-derivative level, or if the Weyl scaling could be corrected by adding higher-derivative terms to maintain the symmetry at higher orders in the curvature expansion, thereby indicating a gauge symmetry of the full bimetric equations. In this paper we investigate this question systematically and, in section 4, show that the corrections to Weyl scaling can be computed at least up to six-derivative terms, equivalent to adding up to 10-derivative corrections to the Bach equation. Before that, in the next section, we consider the relation to partial masslessness and conformal gravity at the quadratic level. 


\section{Linear analysis: PM theory, conformal gravity, and the bimetric model}

The bimetric model specified by (2.14) is precisely the one identified in [18] based on an analysis of partially massless (PM) gauge symmetry in the linearised theory. It propagates six modes (instead of the generic seven) around Einstein backgrounds. The question is if it can lead to a better understanding of partial masslessness at the nonlinear level.

Traditionally, PM symmetry is studied in the context of the linear Fierz-Pauli (FP) theory. Attempts to find nonlinear generalisations are also often influenced by the FP setup, for example, in looking for a fundamental PM field, or in modelling the PM transformations of the nonlinear fields after the linear theory. On the other hand, it is also known that conformal gravity exhibits a spectrum similar to the linear PM theory, except that now, the underlying gauge symmetry is Weyl invariance instead of PM symmetry, and the theory has a spin-2 ghost. After a brief review of these issues, in this section we consider the bimetric model at the quadratic level and show that it provides a unified description of linear PM theory as well as linearised conformal gravity. We argue that the bimetric setup provides a more powerful framework for finding a nonlinear generalisation of PM theory.

\subsection{Partial masslessness in the Fierz-Pauli framework}

The Fierz-Pauli equation for a massive spin-2 field $\delta M_{\mu \nu}$ in a de Sitter metric $\bar{g}_{\mu \nu}$ is,

$$
\mathcal{E}_{\mu \nu}^{\rho \sigma} \delta M_{\rho \sigma}-\Lambda\left(\delta M_{\mu \nu}-\frac{1}{2} \bar{g}_{\mu \nu} \delta M\right)+\frac{1}{2} m_{\mathrm{FP}}^{2}\left(\delta M_{\mu \nu}-\bar{g}_{\mu \nu} \delta M\right)=0,
$$

where the linearised Einstein operator is given by,

$$
\begin{aligned}
(\mathcal{E} \delta M)_{\mu \nu} \equiv-\frac{1}{2}\left(\delta_{\mu}^{\rho} \delta_{\nu}^{\sigma} \bar{\nabla}^{2}+\bar{g}^{\rho \sigma} \bar{\nabla}_{\mu} \bar{\nabla}_{\nu}-\delta_{\mu}^{\rho} \bar{\nabla}^{\sigma} \bar{\nabla}_{\nu}\right. & -\delta_{\nu}^{\rho} \bar{\nabla}^{\sigma} \bar{\nabla}_{\mu} \\
& \left.-\bar{g}_{\mu \nu} \bar{g}^{\rho \sigma} \bar{\nabla}^{2}+\bar{g}_{\mu \nu} \bar{\nabla}^{\rho} \bar{\nabla}^{\sigma}\right) \delta M_{\rho \sigma} .
\end{aligned}
$$

It is well known that when the Higuchi bound is satisfied [42]

$$
m_{\mathrm{FP}}^{2}=\frac{2}{3} \Lambda,
$$

the FP equation becomes invariant under gauge transformations [12],

$$
\Delta\left(\delta M_{\mu \nu}\right)=\left(\nabla_{\mu} \nabla_{\nu}+\frac{\Lambda}{3} \bar{g}_{\mu \nu}\right) \xi(x) .
$$

Consequently, the linear spin-2 field $\delta M_{\mu \nu}$ has four propagating modes, instead of the usual five for a massive field. This is related to the fact that the de Sitter group also has a four-component "partially massless" representation, besides the usual five-component massive representation [11-13]. To take gravity into account, we may add a massless spin-2 field $\delta G_{\mu \nu}$ with two propagating modes, to the linear PM theory.

An interesting question, investigated by many authors [18-20, 26-29, 32, 33, 35, 43], is if the linear PM theory in de Sitter background can be extended to arbitrary backgrounds and if it can be generalised to a nonlinear theory in a background independent way. However, some features of equation (3.1) may be taken as indications that the FP setup is not an adequate starting point for such generalisations: 
1. In the FP setup, there is no analogue of PM theory in flat spacetime, indicating a preferred choice of background. For $\Lambda=0,(3.1)$ and (3.3) describe a massless spin-2 field with two propagating modes. In this case, $\delta M_{\mu \nu}$ cannot be a PM field since, unlike the de Sitter group, the Poincaré group has no four-component spin-2 representation.

2. In a theory with general covariance, $\delta M_{\mu \nu}$ cannot be a fluctuation of some background independent tensor field $M_{\mu \nu}$. This is because (3.1) is not invariant under infinitesimal reparameterisations of $\delta M_{\mu \nu}$. Then, $\delta M_{\mu \nu}$ must the fluctuation of some background dependent (and hence, not fundamental) tensor field $M_{\mu \nu}$ in such a way that, precisely in de Sitter spacetimes, it becomes reparameterisation invariant. ${ }^{7}$ Below we show that these issues can be naturally addressed in a bimetric setup.

\subsection{Linearised conformal gravity and PM in bimetric framework}

Conformal gravity (CG) is defined by the action $\int \mathrm{d}^{4} x \sqrt{g} W^{2}$, where $W_{\mu \nu \rho \sigma}$ is the Weyl curvature tensor. The action is invariant under Weyl scalings of the metric $g \rightarrow e^{\phi} g$. The equation of motion is the Bach equation $B_{\mu \nu}=0$. Due to Weyl invariance, this equation propagates six modes of which two are healthy and four are ghosts (or vice versa, depending on the overall sign of the action). As observed in [7], in a de Sitter background, the linear spectrum is similar to the spectrum of the PM theory with an extra massless spin-2 field, except that one of the fields is now a ghost, and the PM symmetry is replaced by Weyl invariance. This may be taken as a hint of a connection between CG and PM theories. An attempt to identify a nonlinear PM field in CG was made in [43] but no such field was found. Note that unlike the PM theory in the FP framework, conformal gravity admits flat space as a background around which it propagates six modes; two massless spin-2 fields and one massless vector [6].

Now, we consider the bimetric model (2.14) at the quadratic level and show that it provides a unified description of both linear PM theory and linearised conformal gravity. The Einstein-de Sitter solutions in this model are of the type $\bar{f}_{\mu \nu}=c^{2} \bar{g}_{\mu \nu}$ where the equations leave the constant $c^{2}$ arbitrary. The arbitrariness is unique to this model and is a consequence of PM symmetry (see footnote 8 below). In these backgrounds, the fluctuations $\delta g_{\mu \nu}=g_{\mu \nu}-\bar{g}_{\mu \nu}$ and $\delta f_{\mu \nu}=f_{\mu \nu}-\bar{f}_{\mu \nu}$ combine into massless and massive spin-2 modes [40],

$$
\delta G_{\mu \nu}=\delta g_{\mu \nu}+\alpha^{2} \delta f_{\mu \nu}, \quad \delta M_{\mu \nu}=\delta f_{\mu \nu}-c^{2} \delta g_{\mu \nu}
$$

The cosmological constant and Fierz-Pauli mass in this model satisfy the Higuchi bound and are given by (in the background metric $\bar{g}_{\mu \nu}$ and using the notation in (2.5)),

$$
\Lambda_{g}=\frac{3}{2} m_{\mathrm{FP}}^{2}=3 \beta_{2} \mu^{2}\left(\alpha^{-2}+c^{2}\right) .
$$

\footnotetext{
${ }^{7}$ This leaves out the possibility that $\delta M_{\mu \nu}$ is a nonlinear field with some nonlinear completion of (3.1).
} 
The linearised bimetric action, diagonalised into the above mass eigenstates is,

$$
\begin{aligned}
S_{\text {lin }}= & \frac{-m_{g}^{2}}{1+\alpha^{2} c^{2}} \int \mathrm{d}^{4} x\left[\delta G_{\mu \nu} \mathcal{E}^{\mu \nu \rho \sigma} \delta G_{\rho \sigma}-\frac{\Lambda_{g}}{2}\left(\delta G^{\mu \nu} \delta G_{\mu \nu}-\frac{1}{2} \delta G^{2}\right)\right. \\
& \left.+\alpha^{2} c^{-2}\left\{\delta M_{\mu \nu} \mathcal{E}^{\mu \nu \rho \sigma} \delta M_{\rho \sigma}-\frac{\Lambda_{g}}{2}\left(\delta M^{\mu \nu} \delta M_{\mu \nu}-\frac{1}{2} \delta M^{2}\right)+\frac{\Lambda_{g}}{6}\left(\delta M^{\mu \nu} \delta M_{\mu \nu}-\delta M^{2}\right)\right\}\right],
\end{aligned}
$$

where $\mathcal{E}$ is the linear Einstein operator defined in (3.2). This action is invariant under linearised diffeomorphisms of $\delta G_{\mu \nu}$ as well as the linear PM gauge transformation,

$$
\Delta\left(\delta M_{\mu \nu}\right)=A\left(\bar{\nabla}_{\mu} \partial_{\nu}+\frac{\Lambda_{g}}{3} \bar{g}_{\mu \nu}\right) \xi(x)
$$

with gauge parameter $\xi(x)$ and for any $A$. In principle, this may also be accompanied by a (restricted) coordinate transformation of $\delta G_{\mu \nu}$,

$$
\Delta\left(\delta G_{\mu \nu}\right)=B \bar{\nabla}_{\mu} \partial_{\nu} \xi(x)
$$

Using (3.5) one can easily work out the corresponding transformations for the original variables $\Delta\left(\delta g_{\mu \nu}\right)$ and $\Delta\left(\delta f_{\mu \nu}\right){ }^{8}$ Either $A$ or $B$ can be absorbed in a rescaling of $\xi$, as long as it remains non-singular. Beyond that, the arbitrariness in $A$ and $B$ cannot be fixed in the quadratic theory. In section 4.4 we show one can set $B=1$ and nonlinear considerations in bimetric theory determine,

$$
A=\frac{1}{2 \alpha^{2}}\left(1-\alpha^{2} c^{2}\right)
$$

Owing to the above gauge symmetries, $\delta M_{\mu \nu}$ is a partially massless field and $\delta G_{\mu \nu}$ is a massless field, with four and two propagating modes, respectively. For positive values of $c^{2}$, non of the modes is a ghost and (3.7) is a healthy action.

As pointed out in [7], conformal gravity in a de Sitter background has the same structure of modes as above, except that the PM field is a ghost. The reason for this similarity can be easily understood at the quadratic level. For negative values of $c^{2}$ in the action (3.7) the field $\delta M_{\mu \nu}$ becomes a ghost because the sign of its kinetic term changes. In this case the linearised bimetric action can be related to the linearised conformal gravity action. To see this, consider $0>c^{2}>-\alpha^{2}$, and rescale the fluctuations by real constants (with $|c|=\sqrt{-c^{2}}$ ),

$$
\delta M_{\mu \nu} \longrightarrow \sqrt{\frac{6}{\Lambda_{g}}} \frac{|c|}{\alpha m_{g}} \delta M_{\mu \nu}, \quad \delta G_{\mu \nu} \longrightarrow \sqrt{\frac{\Lambda_{g}}{6}} \frac{1}{m_{g}} \delta G_{\mu \nu},
$$

followed by an additional field redefinition,

$$
\delta G_{\mu \nu}^{\prime}=\delta G_{\mu \nu}-\frac{6}{\Lambda_{g}} \delta M_{\mu \nu}
$$

\footnotetext{
${ }^{8}$ As shown in [18], for constant $\xi$, these transformations can be integrated to finite ones only if $c^{2}$ is undetermined. This, in turn, uniquely led to the parameters (2.14). Thus, changes in $c^{2}$ are associated with constant PM "gauge" transformations restricted to the proportional backgrounds. For more general cosmological backgrounds, the equations leave a time-dependent function undetermined [18].
} 
Only for $c^{2}<0$, this replaces the kinetic term of $\delta M_{\mu \nu}$ by a kinetic mixing term,

$$
\begin{aligned}
S_{\text {lin }}^{\prime}= & \frac{1}{1+\alpha^{2} c^{2}} \int \mathrm{d}^{4} x\left[\frac{\Lambda_{g}}{6}\left(-\delta G_{\mu \nu}^{\prime} \mathcal{E}^{\mu \nu \rho \sigma} \delta G_{\rho \sigma}^{\prime}+\frac{\Lambda_{g}}{2}\left(\delta G^{\prime \mu \nu} \delta G_{\mu \nu}^{\prime}-\frac{1}{2} \delta G^{2}\right)\right)\right. \\
& \left.-2 \delta M_{\mu \nu} \mathcal{E}^{\mu \nu \rho \sigma} \delta G_{\rho \sigma}^{\prime}+\Lambda_{g}\left(\delta M^{\mu \nu} \delta G_{\mu \nu}^{\prime}-\frac{1}{2} \delta M \delta G^{\prime}\right)+\delta M^{\mu \nu} \delta M_{\mu \nu}-\delta M^{2}\right]
\end{aligned}
$$

This action is the linearised form of the auxiliary-field formulation of conformal gravity [3],

$$
S_{\text {aux }}=\frac{1}{1+\alpha^{2} c^{2}} \int \mathrm{d}^{4} x \sqrt{G^{\prime}}\left[\frac{\Lambda_{g}}{6}\left(R-2 \Lambda_{g}\right)-M^{\mu \nu}\left(\mathcal{G}_{\mu \nu}+\Lambda_{g} G_{\mu \nu}^{\prime}\right)+M^{\mu \nu} M_{\mu \nu}-M^{2}\right],
$$

where $R$ and $\mathcal{G}_{\mu \nu}$ are the scalar curvature and Einstein tensor of $G_{\mu \nu}^{\prime}$. The solution to the $M_{\mu \nu}$ equation of motion,

$$
M_{\mu \nu}=-\frac{\Lambda_{g}}{6} G_{\mu \nu}^{\prime}+\frac{1}{2}\left(R_{\mu \nu}-\frac{1}{6} G_{\mu \nu}^{\prime} R\right),
$$

when plugged back into (3.14), gives the conformal gravity action for $G_{\mu \nu}^{\prime}$,

$$
S_{\mathrm{C}}=-\frac{1}{4\left(1+\alpha^{2} c^{2}\right)} \int \mathrm{d}^{4} x \sqrt{G^{\prime}}\left(R^{\mu \nu} R_{\mu \nu}-\frac{1}{3} R^{2}\right) .
$$

It is then obvious that integrating out $\delta M_{\mu \nu}$ in (3.13) leads to the linearised form of this action. We emphasise that the manipulations (3.11) and (3.12), remove the $\delta M_{\mu \nu}$ kinetic term only if $c^{2}<0$. Thus, considering the bimetric model in a regime with spin-2 ghosts is crucial for making the connection to conformal gravity at the level of the action.

We conclude that bimetric theory, around its proportional backgrounds, gives a unified description of linearised conformal gravity and linear PM theory. At the quadratic level, both these theories are different phases of the same bimetric model. It should be emphasised that although the undetermined modulus parameter $c^{2}$ is equivalent to a gauge parameter, it is not possible to start with $c^{2}>0$ and reach $c^{2}<0$ by continuous gauge transformations. This is because such transformations would necessarily have to cross $c^{2}=0$, for which one of the metrics becomes singular. Hence, the ghost-free linearised PM theory does not lie on the same gauge orbit as the linearised conformal gravity.

As a final remark, the quadratic bimetric and CG actions are equivalent only for $c^{2}<0$, while for $c^{2}>0$, the actions are not equivalent. However, for both $c^{2}>0$ and $c^{2}<0$, the linearised bimetric equations lead to the linearised Bach equation. For any $c^{2}$, the linearised bimetric equations are,

$$
\begin{aligned}
& \mathcal{E}_{\mu \nu}{ }^{\rho \sigma} \delta g_{\rho \sigma}-\frac{\Lambda_{g}}{2}\left(\delta g_{\mu \nu}-\frac{1}{2} \delta g g_{\mu \nu}\right)-\frac{2 \alpha^{2} \Lambda_{g}}{3\left(1+\alpha^{2} c^{2}\right)}\left(\delta f_{\mu \nu}-c^{2} \delta g_{\mu \nu}-\left(\delta f-c^{2} \delta g\right) g_{\mu \nu}\right)=0 \\
& \mathcal{E}_{\mu \nu}{ }^{\rho \sigma} \delta f_{\rho \sigma}-\frac{\Lambda_{g}}{2}\left(\delta f_{\mu \nu}-\frac{1}{2} \delta f g_{\mu \nu}\right)+\frac{2 \Lambda_{g}}{3\left(1+\alpha^{2} c^{2}\right)}\left(\delta f_{\mu \nu}-c^{2} \delta g_{\mu \nu}-\left(\delta f-c^{2} \delta g\right) g_{\mu \nu}\right)=0
\end{aligned}
$$

Solving the first of these equations for $\delta f_{\mu \nu}$ and plugging the result into the second always gives the linearised Bach equation for $\delta g_{\mu \nu}$,

$$
\frac{\delta B_{\mu \nu}}{\delta g_{\alpha \beta}} \delta g_{\alpha \beta}=0 .
$$




\subsection{Linear theory in flat space}

The discussion in the previous subsection is valid only for $\Lambda_{g} \neq 0$. However, unlike the PM theory in the FP formulation (3.1), the bimetric model also has a flat space solution with six propagating modes around it, without a conflict with the Poincaré group representations. From the expression for $\Lambda_{g}$ (3.6) it is evident that the flat space solution corresponds to a choice of

$$
c^{2}=-\alpha^{-2}
$$

As mentioned above, this value cannot be reached by continuous transformations from $c^{2}>0 . .^{9}$

With the choice (3.20), the expressions of the massless and massive modes in (3.5) coincide or, equivalently, the expressions for $\delta g_{\mu \nu}$ and $\delta f_{\mu \nu}$ in terms of the mass eigenstates become singular. It is therefore not possible to diagonalise the equations in terms of spin-2 mass eigenstates. These are no longer the correct variables to work with, and the earlier discussion of the relation to conformal gravity, formulated in terms of $\delta G_{\mu \nu}$ and $\delta M_{\mu \nu}$, is not valid. This also resolves the conflict with the absence of spin-2 PM representations of the Poincaré group. The equations are, however, well-defined in terms of the original $\delta g_{\mu \nu}$ and $\delta f_{\mu \nu}$ and become most transparent when expressed in terms of $\delta g_{\mu \nu}$ and $\delta M_{\mu \nu}=\delta G_{\mu \nu}=\delta g_{\mu \nu}+\alpha^{2} \delta f_{\mu \nu}$,

$$
\begin{aligned}
\mathcal{E}_{\mu \nu}^{\prime \rho \sigma} \delta M_{\rho \sigma} & =0, \\
\mathcal{E}_{\mu \nu}^{\prime \rho \sigma} \delta g_{\rho \sigma}+\alpha^{-2} \mu^{2} \beta_{2}\left(\delta M_{\mu \nu}-\eta_{\mu \nu} \delta M_{\rho}^{\rho}\right) & =0
\end{aligned}
$$

where $\mathcal{E}_{\mu \nu}^{\prime \rho \sigma}$ is the linearised Einstein operator (3.2) in flat space. Despite the form of the first equation, $\delta M_{\mu \nu}$ is not a massless spin-2 field because the entire system is not invariant under linearised coordinate transformations of $\delta M_{\mu \nu}$. Coordinate transformations of $\delta g_{\mu \nu}$,

$$
\delta g_{\mu \nu} \longrightarrow \delta g_{\mu \nu}+\partial_{\mu} \xi_{\nu}+\partial_{\nu} \xi_{\mu}
$$

are symmetries of (3.21) but $\delta g_{\mu \nu}$ does not satisfy the equation of a massless spin-2 field. The system of perturbation equations is also invariant under a scalar gauge symmetry which transforms both $\delta g_{\mu \nu}$ and $\delta M_{\mu \nu}$,

$$
\Delta \delta g_{\mu \nu}=\frac{\mu^{2} \beta_{2}}{\alpha^{2}} \xi \eta_{\mu \nu}, \quad \Delta \delta M_{\mu \nu}=\partial_{\mu} \partial_{\nu} \xi
$$

Moreover, taking the divergence of (3.21b) shows that $\delta M_{\mu \nu}$ is transverse. Therefore, the number of propagating degrees of freedom around flat background is

$$
\begin{aligned}
20 \text { components } & -2 \cdot 4 \text { coordinate transformations } \\
& -2 \cdot 1 \text { scalar gauge transformations }-4 \text { constraints }=6 .
\end{aligned}
$$

\footnotetext{
${ }^{9}$ Flat space solutions do not exist in the corresponding massive gravity model of [28]. In this case, the cosmological constant of the proportional backgrounds is $\Lambda_{g}=3 c^{2} \mu^{2} \beta_{2}$, where again $c$ is undetermined. Implementing $\Lambda_{g}=0$ by setting $c^{2}=0$ leads to a singular metric (since $f_{\mu \nu}$ is fixed). More generally, in the massive gravity limit, the CG-related phase of the bimetric model as well as many other solutions disappear [31], although more pathological solutions for $c^{2}<0$ could survive.
} 
This is the same number of modes as found around a de Sitter background, where the fluctuations are diagonalisable into a massless and a partially massless field. Around flat space, however, no interpretation in terms of mass eigenstates with spin- 2 alone exist. In fact, the spectrum is the same as that of conformal gravity which consists of two massless spin-2 and one massless spin-1 fields [2, 4-6]. Depending on the overall sign of the action, four or two out of the six propagating modes are ghosts.

\section{A systematic study of the extended Weyl symmetry}

Motivated by the appearance of the Weyl invariant Bach equation at lowest order in the derivative expansion of bimetric equations (2.15), we now investigate the possibility of extending this invariance to higher-derivative terms in the expansion.

\subsection{General form of symmetry transformations}

Let us consider the bimetric model (2.14) in more detail. The action is given by (2.1) with the potential,

$$
V=3 \beta_{2}\left(\alpha^{-2}+\frac{1}{6}\left(\left[S^{2}\right]-[S]^{2}\right)+\alpha^{2} \operatorname{det} S\right),
$$

where $[S] \equiv \operatorname{Tr} S$ and $S=\sqrt{g^{-1} f}$. The $g$ and $f$ equations of motion are,

$$
\begin{aligned}
& \frac{1}{\mu^{2} \beta_{2}} R_{\nu}^{\mu}(g)-\frac{3}{\alpha^{2}} \delta^{\mu}{ }_{\nu}+\left(S^{2}-[S] S\right)^{\mu}{ }_{\nu}=0, \\
& \frac{1}{\mu^{2} \beta_{2}} R_{\nu}^{\mu}(f)-3 \delta^{\mu}{ }_{\nu}+\frac{1}{\alpha^{2}}\left(S^{-2}-\left[S^{-1}\right] S^{-1}\right)^{\mu}{ }_{\nu}=0,
\end{aligned}
$$

in which we have raised the first index with $g^{\mu \nu}$ and $f^{\mu \nu}$, respectively, and used the notation in (2.5). Note that in terms of rescaled variables,

$$
g_{\mu \nu}^{\prime}=\alpha^{-1} g_{\mu \nu}, \quad f_{\mu \nu}^{\prime}=\alpha f_{\mu \nu}
$$

the equations (4.2) take a very symmetric form,

$$
\begin{aligned}
& \hat{\alpha} R_{\nu}^{\mu}\left(g^{\prime}\right)-3 \delta_{\nu}^{\mu}+\left(S^{2}-\left[S^{\prime}\right] S^{\prime}\right)_{\nu}^{\mu}=0, \\
& \hat{\alpha} R_{\nu}^{\mu}\left(f^{\prime}\right)-3 \delta_{\nu}^{\mu}+\left(S^{\prime-2}-\left[S^{\prime-1}\right] S^{\prime-1}\right)_{\nu}^{\mu}=0,
\end{aligned}
$$

in which we have used the further notation (see (2.5))

$$
\hat{\alpha} \equiv \frac{\alpha}{\mu^{2} \beta_{2}}
$$

These transform into each other under the interchange $g_{\mu \nu}^{\prime} \leftrightarrow f_{\mu \nu}^{\prime}$, keeping all parameters fixed. This property will play an important role in the considerations below.

Our aim is to check if we could find nontrivial, field-dependent transformations $g^{\prime} \rightarrow$ $g^{\prime}+\Delta g^{\prime}\left(g^{\prime}, f^{\prime}\right)$ and $f^{\prime} \rightarrow f^{\prime}+\Delta f^{\prime}\left(g^{\prime}, f^{\prime}\right)$ (involving also a scalar gauge parameter) that keep the above equations invariant. Since dealing with the variation $\delta S$ of a square-root matrix is cumbersome, we do not directly study the variations of (4.4), but rather attempt to construct the symmetry perturbatively. First, we deduce some general properties of such transformations. 
Interchange symmetry: The $g^{\prime}$-equation (4.4a) can be rendered invariant for any $\Delta g^{\prime}\left(g^{\prime}, f^{\prime}\right)$ by choosing an appropriate compensating transformation $\Delta f^{\prime}\left(g^{\prime}, f^{\prime}\right)$. Such a $\Delta f^{\prime}$ can be computed for any $\Delta g^{\prime}$ at least perturbatively, for example, by using the perturbative solution for $f^{\prime}$ in terms of $g^{\prime}$ and its curvatures in (2.9). To find a symmetry of both equations, one must be able to specify $\Delta g^{\prime}$ such that the same variations also keep the $f^{\prime}$-equation (4.4b) invariant. Let us assume that such transformations exist.

On the other hand, since the $f^{\prime}$-equation $(4.4 \mathrm{~b})$ is obtained from the $g^{\prime}$-equation by simply interchanging $g^{\prime}$ and $f^{\prime}$, it must also inherit the symmetry of the $g^{\prime}$-equation. In other words, the $f^{\prime}$-equation must also be invariant under,

$$
\Delta_{\text {new }} g^{\prime}=\left.\Delta f^{\prime}\right|_{f^{\prime} \leftrightarrow g^{\prime}}, \quad \Delta_{\text {new }} f^{\prime}=\left.\Delta g^{\prime}\right|_{f^{\prime} \leftrightarrow g^{\prime}}
$$

A similar argument holds for a new symmetry of the $g^{\prime}$-equation. But if we know from various limits that the equations can at most admit one symmetry, then the two sets of transformations must coincide, $\Delta g^{\prime} \equiv \Delta_{\text {new }} g^{\prime}, \Delta f^{\prime} \equiv \Delta_{\text {new }} f^{\prime}$, that is,

$$
\Delta g^{\prime}=\left.\Delta f^{\prime}\right|_{f^{\prime} \leftrightarrow g^{\prime}}, \quad \Delta f^{\prime}=\left.\Delta g^{\prime}\right|_{f^{\prime} \leftrightarrow g^{\prime}}
$$

This property will become very useful in constructing the symmetry transformations.

Field dependence convention: The appearance of the square-root matrix, $S=\sqrt{g^{-1} f}$, in the above equations makes it difficult to investigate their symmetry directly by treating the fields non-perturbatively (although still linearly in the symmetry parameter). Hence here we will work with the derivative expansion of the above equations by recasting the $g^{\prime}$-and $f^{\prime}$-equations as the expansions (2.9) and (2.10) for $f^{\prime}$ and $g^{\prime}$, respectively (see below). Then the gauge transformations can also be expanded as,

$$
\Delta g^{\prime}=\sum_{n=0} \Delta_{(2 n)} g^{\prime}, \quad \Delta f^{\prime}=\sum_{n=0} \Delta_{(2 n)} f^{\prime}
$$

where the variation $\Delta_{(2 n)}$ contains a total of $2 n$ derivatives of the fields and the gauge parameter. In this approach, the expression $\Delta g^{\prime}\left(g^{\prime}, f^{\prime}\right)$ can be expanded in different ways since a dependence on $f^{\prime}$ can be re-expressed in terms of $g^{\prime}$ using (2.9), and vice versa, thereby mixing different orders of curvatures. This ambiguity can be avoided by following the convention that $\Delta g^{\prime}$ is expressed entirely in terms of $g^{\prime}$ and its curvatures, such that $\Delta g^{\prime}=\Delta g^{\prime}\left(g^{\prime}\right)$ and, similarly, $\Delta f^{\prime}=\Delta f^{\prime}\left(f^{\prime}\right)$.

The bootstrap construction: In the previous section we saw that, on perturbatively eliminating $f^{\prime}$ between equations (4.4), one obtains the Bach equation for $g^{\prime}$, plus corrections involving higher powers of $R_{\mu \nu}\left(g^{\prime}\right) / \mu^{2}$. Thus, at the lowest order, $\Delta_{(0)} g_{\mu \nu}^{\prime}=\phi g_{\mu \nu}^{\prime}$ is a symmetry. To check if this symmetry extends to higher orders, a straightforward approach would be compute the higher derivative corrections to the Bach equation and see if corresponding higher order corrections $\Delta_{(2 n)} g^{\prime}$ to the symmetry transformation exit. This would show the existence of an on-shell symmetry in this perturbative framework. The variation $\Delta f^{\prime}$ could 
then be computed by plugging $\Delta g^{\prime}$ in the expansion (2.9) and expressing the outcome entirely in terms of $f^{\prime}$ using (2.10). The $\Delta g^{\prime}$ and $\Delta f^{\prime}$ obtained in this way will automatically satisfy (4.7). In this approach, to compute, for example, the 4-derivative term in $\Delta g^{\prime}$ one needs to know the 8-derivative correction to the Bach equation, which is rather lengthy to compute and manipulate.

Below we follow an alternative bootstrap approach where, starting with $\Delta_{(0)} g^{\prime}$, the transformation is systematically constructed order by order, by using the perturbative expansions for $f^{\prime}$ and $g^{\prime}$ and imposing the interchange symmetry (4.7). The transformations are then guaranteed to leave (4.4) invariant order by order.

\subsection{Structure of the derivative expansion of the bimetric equations}

As discussed in section 2.2, the $g_{\mu \nu}$ equation (4.2a) contains $f_{\mu \nu}$ algebraically through $S_{\nu}^{\mu}$ and hence can be solved perturbatively to express $f_{\mu \nu}$ in terms of $R_{\mu \nu}(g) / \mu^{2} \beta_{2}$. A similar statement holds for the $f_{\mu \nu}$ equation (4.2b). ${ }^{10}$ For the model (4.1), the expressions (2.9) for $f_{\mu \nu}$ and (2.10) for $g_{\mu \nu}$ obtained in this way become,

$$
\begin{aligned}
& f_{\mu \nu}=-\frac{1}{\alpha^{2}} g_{\mu \nu}+\frac{1}{\beta_{2} \mu^{2}} P_{\mu \nu}+4 \sum_{n=2}^{\infty} \frac{\alpha^{2 n-2}}{\left(4 \beta_{2} \mu^{2}\right)^{n}} \gamma_{\mu \nu}^{(2 n)}[g], \\
& g_{\mu \nu}=-\alpha^{2} f_{\mu \nu}+\frac{\alpha^{2}}{\beta_{2} \mu^{2}} \tilde{P}_{\mu \nu}+4 \sum_{n=2}^{\infty} \frac{\alpha^{2}}{\left(4 \beta_{2} \mu^{2}\right)^{n}} \gamma_{\mu \nu}^{(2 n)}[f] .
\end{aligned}
$$

In terms of the rescaled variables (4.3) and (4.5), these take an interchange symmetric form,

$$
\begin{aligned}
& f_{\mu \nu}^{\prime}=-g_{\mu \nu}^{\prime}+\hat{\alpha} P_{\mu \nu}+4 \sum_{n=2}^{\infty}\left(\frac{\hat{\alpha}}{4}\right)^{n} \gamma_{\mu \nu}^{(2 n)}\left[g^{\prime}\right], \\
& g_{\mu \nu}^{\prime}=-f_{\mu \nu}^{\prime}+\hat{\alpha} \tilde{P}_{\mu \nu}+4 \sum_{n=2}^{\infty}\left(\frac{\hat{\alpha}}{4}\right)^{n} \gamma_{\mu \nu}^{(2 n)}\left[f^{\prime}\right] .
\end{aligned}
$$

Here $\gamma_{\mu \nu}^{(2 n)}[g]$ denote terms with $2 n$ derivatives, involving $n$ powers of $P_{\mu \nu}$ and $(n-1)$ powers of the inverse metric $g^{\mu \nu}$ to contract the indices. In appendix B.1, we provide their explicit form for terms up to fifth order in $P_{\mu \nu}$, i.e. up to $n=5$. The same functions $\gamma_{\mu \nu}^{(2 n)}$ appear in both equations due to the $g^{\prime} \leftrightarrow f^{\prime}$ interchange symmetry of the model. Starting from (4.4), one can easily write down recurrence relations that generate $\gamma^{(2 n)}$ to any order. Note that such expansions are not valid in the massive gravity limit $(\alpha \rightarrow \infty)$ of bimetric theory.

\footnotetext{
${ }^{10}$ In other words, equation (4.4a) can be viewed as algebraically determining $S^{\prime}$ in terms of the matrix $\hat{\alpha} R_{\nu}^{\mu}\left(g^{\prime}\right)-3 \delta^{\mu}{ }_{\nu}$, or $\hat{\alpha} P_{\nu}^{\mu}\left(g^{\prime}\right)-\delta^{\mu}{ }_{\nu}$, after subtracting $\frac{\hat{\alpha}}{6} R$. So, in principle, (4.4a) can be re-expressed as,

$$
S^{\prime}=F\left(\hat{\alpha} P_{\nu}^{\mu}\left(g^{\prime}\right)-\delta_{\nu}^{\mu}\right)
$$

where $F$ denotes some matrix function. Since $P_{\nu}^{\mu}$ commutes with $\delta^{\mu}{ }_{\nu}$, for small enough $\hat{\alpha} P_{\nu}^{\mu}, F$ can be expanded in a power series in the usual way. Alternatively, this power series can be obtained directly by perturbatively solving (4.4a) for $S^{\prime}$, which is our approach here. Finally we compute (4.11a) from $f=g S^{2}$.
} 
Equations (4.11) are perturbatively equivalent to the equations of motion (4.4) in the algebraic sense (for the purposes of symmetry arguments that involve algebraic manipulations alone, it is not necessary that they are also equivalent as differential equations). Also, at the lowest orders, the two expansions are compatible. ${ }^{11}$ Finally, we note that all $\gamma_{\mu \nu}^{(2 n)}$ possess the following important properties:

- $\gamma_{\mu \nu}^{(2 n)}$ vanishes identically on Einstein backgrounds for which $P_{\mu \nu} \propto g_{\mu \nu}$.

- Beyond that, $\gamma_{\mu \nu}^{(2 n)}$ vanishes identically on the homogeneous and isotropic solutions of [44] for which, in the above model, $P_{\mu \nu} \propto\left(g_{\mu \nu}+\alpha^{2} f_{\mu \nu}\right)$.

- Linear perturbations of $\gamma_{\mu \nu}^{(2 n)}$ around Einstein backgrounds also vanish identically,

$$
\left.\frac{\delta \gamma_{\mu \nu}^{(2 n)}}{\delta g_{\rho \sigma}}\right|_{\text {Einstein }} \delta g_{\rho \sigma}=0 .
$$

These statements can straightforwardly be proven by noting that for the above type of backgrounds, the complete equations of motion imply $P_{\mu \nu}=\beta_{2} \mu^{2}\left(g_{\mu \nu}+\alpha^{2} f_{\mu \nu}\right)$. But this is saturated by the first two terms in the above expansions, hence the contributions from higher terms must vanish. ${ }^{12}$ This implies that there is no contribution from any of the $\gamma_{\mu \nu}^{(2 n)}$ on these backgrounds. In the same way one verifies the statement about the linear variations.

The above properties imply that for backgrounds of the type $\bar{f}=c^{2} \bar{g}$, all $\gamma^{(2 n)}$ vanish and the expansions in (4.10) or (4.11) become exact. At the lowest order, $\bar{P}_{\mu \nu}=0$ and one gets $\bar{f}=-\bar{g}$. At the quadratic order, $\bar{P}_{\mu \nu}=\left(\Lambda_{g} / 3\right) \bar{g}_{\mu \nu}$ leads to $\bar{f}=c^{2} \bar{g}$, with no higher derivative contributions (with $c^{2}$ and $\Lambda_{g}$ related by (3.6)). At the end of this section we will comment more on the validity regime of these expansions.

\subsection{Perturbative bootstrap construction of gauge symmetry}

Now, employing the criteria collected in section 4.1, we find the condition under which a gauge symmetry could exist and describe a procedure for constructing the transformations to any order. We explicitly show that a gauge symmetry exists to, at least, six orders in derivatives.

Zero-derivative terms: Guided by the Weyl invariance of the Bach equation, which appeared in the analysis of section 2.2, we take the lowest-order gauge transformation of (4.4) to involve an infinitesimal Weyl scaling of $g^{\prime}$. Then, the $g^{\prime}$ equation (4.4a) can always be rendered

\footnotetext{
${ }^{11}$ The purpose of these expansions here is not to accurately compute perturbative corrections, in which case one has to ensure that the two curvature expansions are mutually compatible. The idea rather is that if equations (4.4) admit a gauge symmetry, then the expanded equations (4.11) will exhibit a symmetry of the form described here, irrespective of the details of the compatibility of the two curvature expansions.

${ }^{12} \mathrm{Up}$ to $n=5$, this can be verified directly from the explicit expressions provided in Appendix B.1.
} 
invariant by a compensating transformation of $f^{\prime}$ which can be easily computed perturbatively using the expression (4.11a) for $f^{\prime}$ in terms of $g^{\prime}$. To lowest order in derivatives, this gives,

$$
\Delta_{(0)} g_{\mu \nu}^{\prime}=\phi g_{\mu \nu}^{\prime}, \quad \Delta_{(0)} f_{\mu \nu}^{\prime}=-\phi g_{\mu \nu}^{\prime}
$$

Using (4.11b), $\Delta_{(0)} f_{\mu \nu}^{\prime}$ can be expressed in terms of $f_{\mu \nu}^{\prime}$ alone. Thus to zeroth order in derivatives, one gets,

$$
\Delta_{(0)} g_{\mu \nu}^{\prime}=\phi g_{\mu \nu}^{\prime}, \quad \Delta_{(0)} f_{\mu \nu}^{\prime}=\phi f_{\mu \nu}^{\prime}
$$

Obviously, the lowest-order terms in the transformations satisfy the criterion of interchange symmetry (4.7) and hence must keep both equations (4.4) invariant to this order. It is trivial to see that on ignoring derivative terms the interaction contributions in (4.4) are invariant under such transformations. ${ }^{13}$

Two-derivative terms: Now we describe in detail the construction of the two-derivative terms in the transformation. The procedure generalises to higher orders straightforwardly.

- Step 1: The first step is to determine the structure of all two-derivative terms that are generated by the zero-derivative part of the transformations. Using $\Delta_{(0)} g^{\prime}$ from (4.14) in (4.11a) and retaining terms with up to two derivatives gives the following compensating transformation,

$$
\Delta_{(0)} f_{\mu \nu}^{\prime}+\Delta_{(2)} f_{\mu \nu}^{\prime}=-\phi g_{\mu \nu}^{\prime}-\hat{\alpha} \nabla_{\mu} \partial_{\nu} \phi+\ldots
$$

The dots denote still missing two-derivative terms. The field dependence ambiguity discussed in section 4.1 is fixed by re-expressing $\Delta f^{\prime}$ entirely in terms of $f^{\prime}$. Using the expression (4.11b) for $g^{\prime}$ in terms of $f^{\prime}$, the above transformation at the two-derivative level then becomes $\phi f_{\mu \nu}^{\prime}-\hat{\alpha} \phi \tilde{P}_{\mu \nu}-\hat{\alpha} \tilde{\nabla}_{\mu} \partial_{\nu} \phi$. Since the equations treat $g^{\prime}$ and $f^{\prime}$ on equal footing, it follows that similar two-derivative terms must also appear in $\Delta_{(2)} g^{\prime}$. Hence we take,

$$
\Delta_{(0)} g_{\mu \nu}^{\prime}+\Delta_{(2)} g_{\mu \nu}^{\prime}=\phi g_{\mu \nu}^{\prime}+a_{1} \phi P_{\mu \nu}+a_{2} \nabla_{\mu} \partial_{\nu} \phi
$$

with constants $a_{1}, a_{2}$ to be determined. Now, using this in (4.11a) to recompute $\Delta f_{\mu \nu}^{\prime}$, and once again expressing its $g^{\prime}$-dependence in terms of $f^{\prime}$ gives,

$$
\Delta_{(0)} f_{\mu \nu}^{\prime}+\Delta_{(2)} f_{\mu \nu}^{\prime}=\phi f_{\mu \nu}^{\prime}-\left(a_{1}+\hat{\alpha}\right) \phi \tilde{P}_{\mu \nu}-\left(a_{2}+\hat{\alpha}\right) \tilde{\nabla}_{\mu} \partial_{\nu} \phi
$$

By construction, these leave (4.11a) invariant on-shell up to second order in derivatives. No new two-derivative terms are generated.

\footnotetext{
${ }^{13}$ Of course, the interaction contributions in (4.4) are invariant under $g^{\prime} \rightarrow A g^{\prime}, f^{\prime} \rightarrow A f^{\prime}$, for any invertible matrix $A$, but only the Weyl part of this admits an extension to include derivative terms.
} 
- Step 2: To ensure that the same transformations also keep (4.11b) invariant, it is sufficient to impose the $g^{\prime} \leftrightarrow f^{\prime}$ interchange symmetry (4.7), as argued in section 4.1. Comparing (4.16) and (4.17), one sees that this requires $a_{1}=a_{2}=-\hat{\alpha} / 2$. Hence the transformations that leave both equations in (4.11) or (4.4) invariant at the twoderivative level are,

$$
\begin{aligned}
& \Delta_{(0)} g_{\mu \nu}^{\prime}+\Delta_{(2)} g_{\mu \nu}^{\prime}=\phi g_{\mu \nu}^{\prime}-\frac{\hat{\alpha}}{2}\left(\phi P_{\mu \nu}+\nabla_{\mu} \partial_{\nu} \phi\right) \\
& \Delta_{(0)} f_{\mu \nu}^{\prime}+\Delta_{(2)} f_{\mu \nu}^{\prime}=\phi f_{\mu \nu}^{\prime}-\frac{\hat{\alpha}}{2}\left(\phi \tilde{P}_{\mu \nu}+\tilde{\nabla}_{\mu} \partial_{\nu} \phi\right) .
\end{aligned}
$$

Let us briefly comment on some ambiguities at the two-derivative level that are avoided by the above construction. One can check explicitly that even for arbitrary $a_{1}$ and $a_{2}$, the transformations are a symmetry of (4.11) at the two-derivative level (although this is fixed by the higher-derivative terms). This is due to the fact that at the two-derivative level, a coordinate transformation $\delta x^{\mu}=-a_{2} g^{\prime \mu \nu} \partial_{\nu} \phi$ induces variations $\delta g_{\mu \nu}^{\prime}=2 a_{2} \nabla_{\mu} \partial_{\nu} \phi$ and $\delta f_{\mu \nu}^{\prime}=-2 a_{2} \tilde{\nabla}_{\mu} \partial_{\nu} \phi$. Also, to this order, the equations (4.11) are unchanged under field redefinitions $g^{\prime} \rightarrow g^{\prime}+a_{1} P$ and $f^{\prime} \rightarrow f^{\prime}-a_{1} \tilde{P}$. Hence, $a_{1}$ and $a_{2}$ are not genuine parameters of the new symmetry. In this case, imposing the exchange symmetry (4.7) not only guarantees the invariance of (4.11b), given that (4.11a) is invariant by construction, but it also fixes the coordinate and field redefinition ambiguities.

Extension to higher orders: The construction of the two-derivative terms straightforwardly generalises to higher orders. Two situations arise depending on the number of derivatives $(2 n+2)$ for even or odd $n$. Given transformations $\Delta g^{\prime}$ and $\Delta f^{\prime}$ to $4 m$-derivative order (odd $n$ ), they can always be extended to $(4 m+2)$-derivative order. But starting with $\Delta g^{\prime}$ and $\Delta f^{\prime}$ to $(4 m-2)$-derivative order (even $\left.n\right)$, an extension to $4 m$-derivative order exists only under certain conditions. These need to be satisfied in order for a complete perturbative expression of the gauge symmetry to exist. Remarkably, the four-derivative terms turn out to meet this condition and hence we are able to prove the presence of a gauge symmetry up to six orders in derivatives.

In order to demonstrate the above, let us try to generalise the two-derivative construction to terms with arbitrary number of derivatives in the spirit of a proof by induction. For this we assume that $\sum_{k=0}^{n} \Delta_{(2 k)} g^{\prime}$ and $\sum_{k=0}^{n} \Delta_{(2 k)} f^{\prime}$ are gauge symmetries of (4.11) at the $2 n$ derivative level and satisfy the interchange symmetry (4.7). This implies that if we use $\sum_{k=0}^{n} \Delta_{(2 k)} g^{\prime}$ in (4.11a) and express the resulting variation for $f^{\prime}$ entirely in terms of $f^{\prime}$ using $(4.11 \mathrm{~b})$, then we get $\sum_{k=0}^{n} \Delta_{(2 k)} f^{\prime}$. Moreover, the interchange symmetry insures the invariance of (4.11b).

We are now looking for transformations $\sum_{k=0}^{n+1} \Delta_{(2 k)} g^{\prime}$ and $\sum_{k=0}^{n+1} \Delta_{(2 k)} f^{\prime}$ that keep the equations invariant to next order. The new purely $(2 n+2)$-derivative terms, $\Delta_{(2 n+2)} g^{\prime}$ and $\Delta_{(2 n+2)} f^{\prime}$ can be constructed following the two steps outlined earlier.

- Step 1: We start by finding all relevant $(2 n+2)$-derivative terms, i.e., those that are generated from lower orders. Using $\sum_{k=0}^{n} \Delta_{(2 k)} g^{\prime}$ in (4.11a) to compute the variation 
for $f^{\prime}$ produces directly a set of $(2 n+2)$-derivative terms in terms of $g^{\prime}$. Furthermore, converting all $g^{\prime}$ into $f^{\prime}$ using (4.11b) produces additional terms that will appear in $\Delta_{(2 n+2)} f^{\prime}{ }^{14}$ Motivated by the interchange symmetry $(4.7)$, we then introduce all the corresponding terms with arbitrary coefficients into $\Delta_{(2 n+2)} g^{\prime}$ and recompute the form of $\Delta_{(2 n+2)} f^{\prime}$ from (4.11a). As a representative of the $(2 n+2)$-derivative terms in the transformations, let us consider, for instance, a term of the type $A \phi \tilde{P}_{\mu \nu}^{n+1}$ (for a constant $A$ ) which is generated in $\Delta f^{\prime}$ on using $\Delta g^{\prime}$ at order $2 n$ in (4.11a), and expressing the result in terms of $g^{\prime}$. This term contains $n$ powers of $f^{\prime \mu \nu}$ to contract the indices. The corresponding term must be included in $\Delta_{(2 n+2)} g^{\prime}$ with an arbitrary coefficient $B$,

$$
\Delta_{(2 n+2)} g_{\mu \nu}^{\prime}=B \phi P_{\mu \nu}^{n+1}+\cdots
$$

Now when $\Delta_{(2 n+2)} f^{\prime}$ is recomputed from (4.11a) with $\Delta_{(2 n+2)} g^{\prime}$ included, one gets,

$$
\Delta_{(2 n+2)} f_{\mu \nu}^{\prime}=\left((-1)^{n+1} B+A\right) \phi \tilde{P}_{\mu \nu}^{n+1}+\cdots .
$$

The $A$-term is generated from lower orders, while the $B$-term arises directly from (4.19). The factor of $(-1)^{n+1}$ arises from the replacement $g_{\mu \nu}^{\prime}=-f_{\mu \nu}^{\prime}+\ldots$ in $\phi P_{\mu \nu}^{n+1}$ (we pick up a minus sign for each of the $n$ factors of $g^{\prime \mu \nu}$ ), along with the overall minus sign for computing the transformation of $f^{\prime}$ from the first term of (4.11a). By construction, these transformations leave (4.11a) invariant.

- Step 2: The second step is to impose the interchange symmetry (4.7) in order to insure the invariance of (4.11b). This yields the condition, for any single term,

$$
B=(-1)^{n+1} B-A \text {. }
$$

Clearly, this can be solved for $B$ only for even $n=2 m$. This means that we can impose the interchange symmetry guaranteeing the invariance of both (4.11a) and (4.11b) only for terms with $(4 m+2)$ derivatives. For terms with odd $n=2 m-1$, or $4 m$ derivatives, the construction fails unless $A=0$. This must hold for all types of terms generated at this order. If this condition is met, i.e. if all contributions generated from lower orders cancel each other out, then the coefficients $B$ are left arbitrary by the construction at this order in the transformation, though they may be fixed by higher orders.

We conclude that an on-shell gauge symmetry can be constructed provided that, at each $4 m$ derivative level, the contributions generated from lower orders vanish on-shell. (Dis)proving the existence of a nonlinear gauge symmetry in the equations (4.11) or (4.4) reduces to checking whether this condition is violated or not.

\footnotetext{
${ }^{14}$ Note that all terms with lower number of derivatives are already accounted for in $\sum_{k=0}^{n} \Delta_{(2 k)} f^{\prime}$.
} 
Four- and six-derivative terms: The above analysis shows that, in this approach, an obstruction first arises at the four-derivative level. For a gauge symmetry to exist, it is necessary that the variation $\Delta_{(0)} g^{\prime}+\Delta_{(2)} g^{\prime}$ used in (4.11a) does not generate any fourderivative terms in $\Delta f^{\prime}$. This is not obvious at first sight. However, the outcome of a rather lengthy calculation outlined in appendix B is that such four-derivative terms are indeed not generated by terms with fewer derivatives (this can be easily verified for a constant gauge parameter $\phi$ ). Therefore, the four-derivative terms in the transformations remain arbitrary at this level. They may need to be fixed when considering the eighth-order contributions, which again need to vanish on-shell due to (4.21).

The above construction also shows that, once a symmetry at the four-derivative level is established, invariance at the six-derivative level is guaranteed. Hence we are able to perturbatively construct gauge transformations of (4.11) up to sixth order in derivatives,

$$
\begin{aligned}
& \Delta g_{\mu \nu}^{\prime}=\Delta_{(0)} g_{\mu \nu}^{\prime}+\Delta_{(2)} g_{\mu \nu}^{\prime}+\Delta_{(4)} g_{\mu \nu}^{\prime}+\Delta_{(6)} g_{\mu \nu}^{\prime}+\ldots \\
& \Delta f_{\mu \nu}^{\prime}=\Delta_{(0)} f_{\mu \nu}^{\prime}+\Delta_{(2)} f_{\mu \nu}^{\prime}+\Delta_{(4)} f_{\mu \nu}^{\prime}+\Delta_{(6)} f_{\mu \nu}^{\prime}+\ldots
\end{aligned}
$$

The result for the zero- and two-derivative terms is given in (4.18). Terms with four derivatives in the transformations are completely arbitrary at this stage; any choice will leave the equations invariant up to sixth order in derivatives. The six-derivative terms, $\Delta_{(6)} g^{\prime}$ and $\Delta_{(6)} f^{\prime}$, are again determined by the construction described above (but are expected to depend on the choice of the four-derivative terms). The explicit expressions are tedious, but the derivation is straightforward. ${ }^{15}$ We emphasise that these transformations are covariant and background independent to the extent that the perturbative expansion is valid.

\subsection{Relation to the partially massless gauge symmetry}

In section 3.2 we described how the linear PM theory is easily embedded in a covariant bimetric setup. In this section, we have constructed transformations (4.22), at least up to 6 orders in derivatives, that keep the bimetric equations invariant. In the perturbative setup employed, the transformations are simply generalisations of the Weyl scalings of the metrics augmented by higher derivative corrections. This construction required no input from the PM properties of the quadratic theory.

It is now easy to verify that the linear PM transformations (3.8), (3.9) are recovered from (4.22) at the two-derivative level. This also fixes the arbitrariness of the quadratic theory. In terms of the unscaled metrics $g$ and $f$, the two-derivative variations (4.18) can be rewritten as (after adding and subtracting $\Lambda_{g}$ contributions using (3.6)),

$$
\begin{aligned}
& \Delta g_{\mu \nu}=\frac{1}{2}\left(1-\alpha^{2} c^{2}\right) \phi g_{\mu \nu}-\frac{\alpha^{2}}{2 \mu^{2} \beta_{2}}\left(\phi\left[P_{\mu \nu}-\frac{\Lambda_{g}}{3} g_{\mu \nu}\right]+\nabla_{\mu} \partial_{\nu} \phi\right), \\
& \Delta f_{\mu \nu}=\frac{1}{2}\left(1-\alpha^{-2} c^{-2}\right) \phi f_{\mu \nu}-\frac{1}{2 \mu^{2} \beta_{2}}\left(\phi\left[\tilde{P}_{\mu \nu}-\frac{\Lambda_{g}}{3 c^{2}} f_{\mu \nu}\right]+\tilde{\nabla}_{\mu} \partial_{\nu} \phi\right),
\end{aligned}
$$

\footnotetext{
${ }^{15}$ To find these transformations using corrections to the Bach equation, we would have had to compute up to 10-derivative corrections to the Bach equation.
} 
where, higher derivative terms have been suppressed. Let us restrict the nonlinear fields $g_{\mu \nu}$ and $f_{\mu \nu}$ to $g_{\mu \nu}=\bar{g}_{\mu \nu}+\delta g_{\mu \nu}$ and $f_{\mu \nu}=\bar{f}_{\mu \nu}+\delta f_{\mu \nu}$. The fixed background satisfies $\bar{f}_{\mu \nu}=c^{2} \bar{g}_{\mu \nu}$, so the transformation only affects the fluctuations, $\Delta g_{\mu \nu}=\Delta\left(\delta g_{\mu \nu}\right)$ and $\Delta f_{\mu \nu}=\Delta\left(\delta f_{\mu \nu}\right)$. For such a background, $\bar{P}_{\mu \nu}=\overline{\tilde{P}}_{\mu \nu}=\left(\Lambda_{g} / 3\right) \bar{g}_{\mu \nu}$. Since the transformations are already linear in the small gauge parameter $\phi$, only the background pieces contribute to the right-hand-sides of (4.23). Then, at two-derivative level, the symmetry transformations reduce to,

$$
\begin{aligned}
& \Delta\left(\delta g_{\mu \nu}\right)=\frac{1}{2}\left(1-\alpha^{2} c^{2}\right) \phi \bar{g}_{\mu \nu}-\frac{\alpha^{2}}{2 \beta_{2} \mu^{2}} \bar{\nabla}_{\mu} \partial_{\nu} \phi \\
& \Delta\left(\delta f_{\mu \nu}\right)=-\frac{1}{2 \alpha^{2}}\left(1-\alpha^{2} c^{2}\right) \phi \bar{g}_{\mu \nu}-\frac{1}{2 \beta_{2} \mu^{2}} \bar{\nabla}_{\mu} \partial_{\nu} \phi .
\end{aligned}
$$

For the mass eigenstates (3.5), this implies,

$$
\begin{aligned}
& \Delta\left(\delta M_{\mu \nu}\right)=\frac{\left(\alpha^{2} c^{2}-1\right)}{2 \mu^{2} \beta_{2}}\left(\bar{\nabla}_{\mu} \bar{\nabla}_{\nu}+\frac{\Lambda_{g}}{3} \bar{g}_{\mu \nu}\right) \phi, \\
& \Delta\left(\delta G_{\mu \nu}\right)=-\frac{\alpha^{2}}{\mu^{2} \beta_{2}} \bar{\nabla}_{\mu} \bar{\nabla}_{\nu} \phi
\end{aligned}
$$

This recovers the standard PM transformation of the quadratic theory in Einstein-de Sitter backgrounds, (3.8), along with a coordinate transformation of (3.9) (for $\alpha^{2} \phi=-\mu^{2} \beta_{2} \xi$ ). It also fixes the ambiguity present in the quadratic theory. Note that $\Delta M_{\mu \nu}$ is invariant under infinitesimal coordinate transformations of $g$ and $f$.

In this approach, it is obvious that the standard form of the PM transformation in terms of $\delta M_{\mu \nu}$ is very specific to Einstein-de Sitter backgrounds. Away from such backgrounds, the fundamental fields are $g_{\mu \nu}$ and $f_{\mu \nu}$, and a fundamental PM field does not exist. Furthermore, the nonlinear transformations induce no linear variation of $\delta M_{\mu \nu}$ when $\alpha^{2} c^{2}=1$. We will comment on an implication of this in the next section.

\subsection{Limitations of the perturbative expansion}

The perturbative expansions we have used are valid for small $R_{\mu \nu}(g) /\left(\beta_{2} \mu^{2}\right)$, or small $\hat{\alpha} P_{\mu \nu}\left(g^{\prime}\right)$, where $1 / \beta_{2} \mu^{2}$ or $\hat{\alpha}$ are the small expansion parameters. To connect to the linear PM theory, we considered the expansions around Einstein-de Sitter (EdS) solutions for which $\bar{f}^{\prime}=\alpha^{2} c^{2} \bar{g}^{\prime}$. But these backgrounds have a curvature scale $\Lambda_{g}^{\prime} \sim 1 / \hat{\alpha}$ (as implied by (3.6) in terms of rescaled variables). Thus, in these cases,

$$
\hat{\alpha} P_{\mu \nu}\left(\bar{g}^{\prime}\right)=\left(1+\alpha^{2} c^{2}\right) \bar{g}_{\mu \nu}^{\prime}
$$

is not suppressed by $\hat{\alpha}$ and is small only when $c^{2}$ is close to $-\alpha^{-2}$. In spite of this, the standard $\mathrm{PM}$ results were recovered for any $c^{2}$ from the first two terms in the expansions. The reason is that although now the 4- and higher-derivative terms $\gamma_{\mu \nu}^{(2 n)}\left(\bar{g}^{\prime}\right)$ are not suppressed by $\hat{\alpha}$, they nonetheless vanish on EdS backgrounds and, hence, are small in the vicinity of it, for reasons having to do with the structure of the equations. The linear variations of $\gamma_{\mu \nu}^{(2 n)}$ around EdS solutions also vanish. But, now the expansion in $\gamma_{\mu \nu}^{(2 n)}$ is not perturbative since terms with different $n$ may make small, but comparable contributions. Hence the expansions we have used are definitely reliable as perturbative expansions when $\left|1+\alpha^{2} c^{2}\right|<1$, in which case 
the spectrum of perturbations is not unitary. However, if the presence of a symmetry could be established to all orders in this regime, this would also imply the invariance of the full equations of motion. For $c^{2}>0$, the status of the expansion is less obvious although the linear results around EdS solutions are easily reproduced. In any case, the possibility of extending the symmetry beyond the four-derivative Bach equation clearly points to additional structure within the nonlinear equations.

It is easy to rewrite equations (4.4) such that they are valid perturbative expansions around general proportional backgrounds, but this is not useful for finding the transformations. In (4.4) we can simply rewrite $R_{\mu \nu}\left(g^{\prime}\right)$ as $\left(R_{\mu \nu}\left(g^{\prime}\right)-\Lambda_{g}^{\prime} g_{\mu \nu}^{\prime}\right)+\Lambda_{g}^{\prime} g_{\mu \nu}^{\prime}$ and expand $S^{\prime}$ in powers of $\hat{\alpha}\left(R_{\mu \nu}\left(g^{\prime}\right)-\Lambda_{g}^{\prime} g_{\mu \nu}^{\prime}\right)$, or equivalently, in powers of $\hat{\alpha} \mathcal{P}_{\mu \nu}\left(g^{\prime}\right)=\hat{\alpha}\left(P_{\mu \nu}\left(g^{\prime}\right)-\left(\Lambda_{g}^{\prime} / 3\right) g_{\mu \nu}^{\prime}\right)$. In the notation of footnote 10, the equations imply that $S^{\prime}$ must have the form,

$$
S^{\prime}=F\left(\hat{\alpha} \mathcal{P}_{\nu}^{\mu}+\left(\hat{\alpha} \frac{\Lambda_{g}^{\prime}}{3}-1\right) \delta^{\mu}{ }_{\nu}\right)=F\left(\hat{\alpha} \mathcal{P}_{\nu}^{\mu}+\alpha^{2} c^{2} \delta^{\mu}{ }_{\nu}\right)
$$

where $F(X)$ is a matrix function of the matrix $X$ and traces of powers of $X$. Since $\mathcal{P}_{\nu}^{\mu}$ commutes with $\delta_{\nu}^{\mu}$, for small enough $\hat{\alpha}$, this can be expanded in a power series in the usual way. In practice, this is again achieved by solving the equation perturbatively for $S^{\prime}$. The two lowest-order terms are just rewritings of the corresponding terms in (4.11), $f_{\mu \nu}^{\prime}=\alpha^{2} c^{2} g_{\mu \nu}^{\prime}+$ $\hat{\alpha} \mathcal{P}_{\mu \nu}\left(g^{\prime}\right)+\cdots$. At higher orders, the $\gamma^{(2 n)}$ are now functions of $\mathcal{P}$, rather than $P$, and the coefficients are $c$-dependent. Close to EdS backgrounds, $\hat{\alpha} \mathcal{P}_{\mu \nu}$ are small and the expansion is perturbative. However, under a variation, $\hat{\alpha} \Delta \mathcal{P}_{\mu \nu}=\hat{\alpha} \Delta P_{\mu \nu}-\left(1+\alpha^{2} c^{2}\right) \Delta g_{\mu \nu}^{\prime}$, note that $\Delta g^{\prime}$ is not suppressed by a small $\hat{\alpha}$. Hence higher terms contribute to the variation of lower terms and the expansion is not useful for finding the transformations order by order.

Alternatively, in (4.4) we can rewrite $P_{\mu \nu}\left(g^{\prime}\right)$ as $\left(P_{\mu \nu}-\frac{1}{3} \Lambda_{g}^{\prime} \bar{g}_{\mu \nu}^{\prime}\right)+\frac{1}{3} \Lambda_{g}^{\prime} \bar{g}_{\mu \nu}^{\prime}$. Then,

$$
S^{\prime}=F\left(\hat{\alpha} \hat{\mathcal{P}}_{\nu}^{\mu}+\left(1+\alpha^{2} c^{2}\right) g^{\prime \mu \lambda} \bar{g}_{\lambda \nu}^{\prime}-\delta_{\nu}^{\mu}\right)
$$

Now, taking $\Delta \bar{g}=0$, the variation of $\hat{\mathcal{P}}_{\mu \nu}=P_{\mu \nu}-\frac{1}{3} \Lambda_{g}^{\prime} \bar{g}_{\mu \nu}^{\prime}$ does not mix terms of different order. But $\hat{\mathcal{P}}_{\mu \nu}$ does not commute with $\left(g^{\prime-1} \bar{g}^{\prime}\right)^{\mu}{ }_{\nu}$ and the expansion is involved. Hence there is no easy way of improving over the expansions considered here.

\section{Discussion}

In this section we first discuss our results and then argue that the bimetric model considered here avoids the counter arguments presented so far for the absence of a gauge symmetry in the bimetric setup, at least in their present form.

\subsection{Summary and discussion of results}

In this paper we investigated a possible gauge symmetry of the equations of motion of a particular bimetric model specified by (2.14). The equations contain the square-root matrix

$S=\sqrt{g^{-1} f}$ and the expression for the variation of $S$ in terms of the variations $\Delta g$ and 
$\Delta f$ is complicated. We avoid this complication by working with appropriate perturbative expansions of the equation.

In a small curvature regime, we perturbatively eliminate one of the metrics, say, $f_{\mu \nu}$ between the two equations to get a higher derivative equation for $g_{\mu \nu}$. At the lowest order, instead of the Einstein equation for $g_{\mu \nu}$, for this particular model one obtains the 4-derivative Bach equation of conformal gravity which is invariant under Weyl scalings of $g_{\mu \nu}$. In a systematic treatment, we show that the Weyl scalings of $g_{\mu \nu}$ and $f_{\mu \nu}$ can be corrected at least up to 6-derivative terms to maintain the gauge symmetry at higher orders. A brute force computation of the same transformations, by demanding the invariance of the higherderivative extension of the Bach equation, would have required calculating up to 10-derivative corrections to the Bach equation. Furthermore, we find a sufficiency condition for extending the symmetry to higher orders: In our approach, at orders $4 m$ there are obstructions to the symmetry which must vanish. Then a symmetry at levels $4 m+2$ is insured to exist. Once an obstruction vanishes at a given order $4 m$, the transformations at that level remain completely arbitrary and will be fixed at order $4(m+1)$.

The above results are completely independent of de Sitter backgrounds and linear partial masslessness. Nevertheless, it turns out that in Einstein-de Sitter spacetimes, the lowest-order terms in the transformations do reduce to the well-known PM gauge transformations for a composite PM field. We also considered the bimetric model at the quadratic level and showed that it provides a unified description of both linear PM theory as well as linearised conformal gravity, including in flat spacetime. We argued that the bimetric setup provides a natural and more promising framework for finding a nonlinear PM theory, without a fundamental PM field, as compared to the usual Fierz-Pauli framework.

Our analysis of the transformations as extensions of Weyl scaling is intimately connected to the $g^{\prime} \leftrightarrow f^{\prime}$ interchange symmetry of the equations and breaks down in the massive gravity limit of the theory. Also the equations breakdown for the relevant bimetric theory in 3 dimensions. Such two-derivative theories do not exist in other dimensions. Thus the analysis indicates the absence of such a symmetry in $d=3$, as well as in massive gravity.

If the 6-derivative transformation of the equations of motion found here can be extended to all orders, this would imply a local gauge invariance of the original equations. In the present approach going beyond the 6-derivative order is cumbersome, so the analysis is not conclusive. However, it is evident from our findings that the equations in this bimetric model possess a lot of hidden structure. A possibility is that a gauge symmetry exists and a better understanding of the structure of equations may enable one argue for its presence and even find its closed form expression. The model would then propagate six instead of seven degrees of freedom around any background and would become a ghost-free replacement for conformal gravity. In this case, imbedding the model in a larger setup should make the symmetry manifest.

It is also possible that the equations do not have a gauge symmetry and our construction cannot be extended to all orders. Nonetheless, the results show that the bimetric framework is more powerful than the Fierz-Pauli framework in searching for a nonlinear PM theory. It is 
also possible that a gauge symmetry exists in expected completions of the model. It is obvious that the bimetric model is the spin-2 analogue of the Proca theory in curved background. Hence, just like Proca theory, one may need additional degrees of freedom to obtain the model through a Higgs-like mechanism. This or other extensions may be better candidates for finding a new gauge symmetry. If such a nonlinear PM theory exists it would be very interesting to consider it as an alternative for conformal gravity in the proposal of $[45,46]$, which attempts to embed the Standard Model in a renormalisable theory of gravity.

Finally, the symmetry we have found (at 6-derivative level) is an on-shell symmetry of the equations of motion, except for the quadratic theory, where it is also an off-shell symmetry of the action. In terms of the nonlinear fields, even the constant scalings of the metrics at the zero derivative level are not symmetries of the action, whereas they trivially keep the equations of motion invariant. Of course, off-shell symmetries of the action always imply invariance of the equations of motion, but the converse is not always true.

\subsection{Discussion of the counter-arguments}

Recently, some arguments have been made against the existence of a scalar gauge symmetry in the bimetric model with parameters (2.14). We have shown that this model goes beyond other similar constructions, in particular in displaying PM and conformal gravity behaviours in different phases, but we do not make any claims beyond what we have explicitly computed. It is also interesting to see that the model can evade the counter arguments presented so far and could suggest ways of improving them. First of all, since the transformations we construct are on-shell invariances, any off-shell analysis at the level of the action must also remain valid on-shell to be relevant.

Massive gravity based arguments: The main argument for the absence of a gauge symmetry in the bimetric model is based on the corresponding massive gravity limit. The massive gravity model, obtained in the $\alpha \rightarrow \infty$ limit [47] of the bimetric model (2.14) (for the unscaled

variables $g, f)$, was studied in [27-29]. It also admits proportional backgrounds $g=c^{-2} \bar{f}$ with arbitrary $c^{2}$, where $\bar{f}$ is now a fixed Einstein-de Sitter metric with $\Lambda_{g}=3 \beta_{2} \mu^{2} c^{2}$. The linear theory around this background is precisely the linear PM theory reviewed in section 3.1. Arguments were presented in [27-29] that, in this case, the linear PM symmetry does not extend to the nonlinear theory. It was also argued that since massive gravity can be obtained as a limit of bimetric theory, the absence of a PM symmetry in the massive gravity model implies the same for the bimetric model [27-29].

This argument assumes that the bimetric gauge transformations have an $\alpha \rightarrow \infty$ limit. The results in [20] and in the present paper indicate that this is not the case, as was explained in [31] in detail. We summarise the key points here.

(i) Our derivation of the symmetry to sixth order heavily depended on the $g^{\prime} \leftrightarrow f^{\prime}$ interchange symmetry of the equations, which is destroyed in the massive gravity limit. 
(ii) Our starting point was that the $g$-equation of motion can always be rendered invariant by an appropriate transformation of $f$. In massive gravity, $f$ is a fixed metric that cannot be varied (not even within a class of solutions of the Einstein equation for $f$ ).

(iii) The expansion (4.10a) does not exist in massive gravity, since a term with $2 n$ derivatives diverges as $\alpha^{2 n-2}$ in the limit and there are infinitely many terms with increasing powers of $\alpha$. Hence the massive gravity equations are not writable as the Bach equation plus corrections and the Weyl invariance at the lowest-order in the derivative expansion is not obtainable. Massive gravity therefore lacks the remarkable structure that exists in the bimetric equations.

(iv) The gauge transformations $\Delta g, \Delta f$ obtained from (4.10) or (4.11) also involve an expansion powers of $\alpha$ and hence diverge in the massive gravity limit. The solutions that are obtained by applying these transformations also do not exit in the massive gravity limit. This indicates that these gauge transformations indeed disappear in the massive gravity limit. Of course, these statements are based on a perturbative expansion which, in principle, could sum up into a closed expression with a well defined $\alpha \rightarrow \infty$ limit. But, as pointed out in [31], solutions that are singular in the limit do exist. The key point is that massive gravity is a limit of bimetric theory around specific classes of solutions that need to be specified before taking the limit (a massive gravity limit of the entire bimetric theory does not exist). As discussed in [31], for any solution with a well-defined massive gravity limit, there exists another solution which becomes singular in this limit. ${ }^{16}$

If now a gauge transformation in nonlinear bimetric theory connects these two types of solutions, the perturbative expansion for the transformations will contain an infinite number of increasing powers of $\alpha$ (as is the case for the transformations constructed here), then clearly it cannot survive the massive gravity limit and the corresponding solutions are also absent after the limit has been taken. The form of the transformations derived here suggests that, generically, every gauge orbit contains one solution around which the bimetric theory has a massive gravity limit and the rest of the gauge orbit is invisible in the massive gravity limit.

(v) So far we have argued that if the bimetric model (2.14) has a gauge symmetry of the type discussed here, the symmetry will not survive in the massive gravity limit. But this argument must exclude the PM symmetry of linearised massive gravity around EdS backgrounds. Indeed, in our analysis, the terms that diverge as $\alpha \rightarrow \infty$ are $\gamma_{\mu \nu}^{(2 n)}$ for $n \geq 2$. As mentioned earlier, on EdS backgrounds, $P_{\mu \nu}(\bar{g})=\left(\Lambda_{g} / 3\right) \bar{g}_{\mu \nu}$, both $\gamma_{\mu \nu}^{(2 n)}(\bar{g})$ as well as their first variations vanish (4.12). Hence in this case the transformations do have a massive gravity limit, as should be the case.

Symmetry algebra arguments: Some recent interesting works have studied the restrictions imposed on nonlinear PM theories by the closure of the PM symmetry algebras [32, 35]. Reference [32] considered a general action for a PM field $\phi_{\mu \nu}$ interacting with a metric $G_{\mu \nu}$ and analysed the theory to cubic order in $\phi_{\mu \nu}$. At the lowest order, $G_{\mu \nu}$ was assumed to be invariant and $\phi_{\mu \nu}$ transformed as a linear PM field, but now in a general background. $\phi_{\mu \nu}$-dependent corrections to the transformations were determined from the invariance of the

\footnotetext{
${ }^{16} \mathrm{An}$ example is the flat bimetric solution in section 3.3 that is singular in the massive gravity limit
} 
quadratic and cubic $\phi_{\mu \nu}$ terms. On imposing the closure of the algebra it was found that whenever the PM field had a positive kinetic term, the cubic interactions were imaginary, while real cubic interactions were associated with a ghost PM field (as in conformal gravity). This lead to the conclusion that a unitary interacting theory with PM symmetry does not exist. [32] also emphasised that the action studied covered bimetric theory. If true, this would imply that the symmetry, at the 6-derivative level, found here is an artefact of the $c^{2}<0$ regime of bimetric theory and cannot be extended to higher orders. However, besides the analysis being off-shell, there is a potential caveat. To show that the PM interactions considered also covered bimetric theory, [32] used the relations,

$$
G_{\mu \nu}=g_{\mu \nu}+f_{\mu \nu}, \quad \phi_{\mu \nu}=g_{\mu \nu}-f_{\mu \nu} .
$$

and re-expressed the bimetric action (for $m_{g}=m_{f}$ ) in terms of $G_{\mu \nu}$ and $\phi_{\mu \nu}$. Due to the symmetry $S(g, f)=S(f, g)$ there will be no terms linear in $\phi_{\mu \nu}$ (as assumed in [32]), but also there will be no cubic $\phi_{\mu \nu}$ terms that were crucial in the analysis of [32]. Moreover, the identification of a PM field in bimetric theory is background dependent and the $\phi_{\mu \nu}$ in (5.1) can be identified with a PM field only around proportional backgrounds with $c^{2}=1$. Then, since $\alpha=1$, from the equations in section 4.4 it is evident that around proportional backgrounds, (corresponding to $\phi_{\mu \nu}=0$ ), the PM field does not transform. Note that in this case, the quadratic theory still has an accidental linear PM symmetry, but this does not follow from the nonlinear PM symmetry. The vanishing of the transformation for $c^{2}=1$ is completely consistent with the absence of the cubic $\phi_{\mu \nu}$ interactions. Hence, at least using the identifications (5.1) advertised in [32], the analysis there is avoided by the bimetric model.

Another general study of PM algebra was carried out in [35]. The analysis involved only one dynamical spin-2 field but, nevertheless, seems related to our work. The authors considered general nonlinear extensions of the PM symmetry in dS and, by demanding closure of the transformation algebra, found a unique candidate transformation. The form of this symmetry, and the fact that it had a non-trivial flat space limit, led the authors to the conclusion that such an invariance could not be realised in any two-derivative single-field Lagrangian. These results are fully consistent with ours. In order to compare our setup to the single-field analysis of [35] we have to consider the single-field higher derivative equation (2.15). We found a candidate symmetry of those equations up to six-derivative order (in the transformation), which furthermore also had a flat space counterpart. It is obvious that the single-field equation (2.15) does not derive from any two-derivative single-field action. In fact, as a two-derivative theory they follow from the bimetric action containing both $g_{\mu \nu}$ and $f_{\mu \nu}$ whereas, when viewed as a single-field theory, they are higher-derivative equations which derive from a non-local action obtained after properly integrating out $f_{\mu \nu}$.

Finally, we should mention the constraint analysis of [33] in the $d=3$ version of the present bimetric model given in [19]. The outcome, that the model in 3-dimensions does not posses PM symmetry is consistent with the fact that the expansions in (4.11) do not exist in the $d=3$ theory. 
It is interesting to see if a more refined version of the above analysis could make a more definitive statement about PM theory in the bimetric framework.

Acknowledgments: We thank Luis Apolo, Latham Boyle, Cedric Deffayet, Bo Sundborg, Andrew Waldron and Nico Wintergerst for helpful discussions. The work of ASM is supported by ERC grant no. 615203 under the FP7 and the Swiss National Science Foundation through the NCCR SwissMAP. The research of MvS has received funding from the European Research Council under the European Community's Seventh Framework Programme (FP7/2007-2013 Grant Agreement no. 307934).

\section{A Details of bimetric theory and its curvature expansion}

\section{A.1 Structure of action and equations of motion}

The elementary symmetric polynomials that enter the ghost-free bimetric action can be defined through the following recursion formula,

$$
e_{n}(S)=\frac{1}{n} \sum_{k=0}^{n-1}(-1)^{k+n+1} \operatorname{Tr}\left[S^{n-k}\right] e_{k}(S), \quad e_{0}(S)=1
$$

Note that $e_{4}(S)=\operatorname{det}(S)$ and $e_{n}(S)=0$ for $n>4$, if $S$ is a $(4 \times 4)$-matrix. Explicit expressions can easily be written down. The contributions from the interaction potential to the bimetric equations of motions are given by [23],

$$
\begin{aligned}
& V_{\mu \nu} \equiv-\frac{2}{\sqrt{g}} \frac{\partial(\sqrt{g} V)}{\partial g^{\mu \nu}}=g_{\mu \rho} \sum_{n=0}^{3} \beta_{n}\left[Y^{(n)}(S)\right]^{\rho}{ }_{\nu}, \\
& \tilde{V}_{\mu \nu} \equiv-\frac{2}{\sqrt{f}} \frac{\partial(\sqrt{g} V)}{\partial f^{\mu \nu}}=f_{\mu \rho} \sum_{n=0}^{3} \beta_{4-n}\left[Y^{(n)}\left(S^{-1}\right)\right]_{\nu}^{\rho} .
\end{aligned}
$$

in which we have used the definitions,

$$
\left[Y^{(n)}(S)\right]_{\nu}^{\rho} \equiv \sum_{k=0}^{n}(-1)^{k} e_{k}(S)\left[S^{n-k}\right]_{\nu}^{\rho} .
$$

Since the matrices $S^{-1}$ and $S$ with indices raised or lowered by either of the two metrics are symmetric, the same holds for the $\left[Y^{(n)}(S)\right]^{\rho}{ }_{\nu}$. Note also the following identity,

$$
\left[Y^{(4)}(M)\right]_{\nu}^{\rho}=0
$$

which holds for any $4 \times 4$ matrix $M$ and follows directly from the Cayley-Hamilton theorem. 


\section{A.2 Obtaining the curvature expansions}

The explicit expression for th perturbative solution for $f_{\mu \nu}$ obtained from the $g_{\mu \nu}$ equation $(2.4 \mathrm{a})$ reads [20],

$$
f_{\mu \nu}=a^{2} g_{\mu \nu}+\frac{2 a^{2}}{s_{1} \mu^{2}} P_{\mu \nu}+\frac{a^{2}\left(s_{1}+2 s_{2}\right)}{s_{1}^{3} \mu^{4}} P_{\mu \nu}^{2}+\frac{2 a^{2} s_{2}}{s_{1}^{3} \mu^{4}}\left[\frac{1}{3} e_{2}(P) g_{\mu \nu}-P P_{\mu \nu}\right]+\mathcal{O}\left(\frac{P^{3}}{\mu^{6}}\right),
$$

where $\mu^{2}=m^{4} / m_{g}^{2}$ and all indices on the right-hand side are contracted with $g_{\mu \nu}$. The $s_{n}$ are particular combinations of bimetric parameters given by,

$$
s_{n}=\sum_{k=n}^{3}\left(\begin{array}{l}
3-n \\
k-n
\end{array}\right) a^{k} \beta_{k} .
$$

The value of the coefficient $a^{2}$ is obtained by solving the equation $s_{0}=0$ for $a$ (where we only consider solutions that result in real values for $a^{2}$ ). The solution for $g_{\mu \nu}$ obtained from the $f_{\mu \nu}$ equation $(2.4 \mathrm{~b})$ has the very similar form,

$$
g_{\mu \nu}=\tilde{a}^{2} f_{\mu \nu}+\frac{2 \tilde{a}^{2}}{\tilde{s}_{1} \tilde{\mu}^{2}} \tilde{P}_{\mu \nu}+\frac{\tilde{a}^{2}\left(\tilde{s}_{1}+2 \tilde{s}_{2}\right)}{\tilde{s}_{1}^{3} \tilde{\mu}^{4}} \tilde{P}_{\mu \nu}^{2}+\frac{2 \tilde{a}^{2} \tilde{s}_{2}}{\tilde{s}_{1}^{3} \tilde{\mu}^{4}}\left[\frac{1}{3} e_{2}(\tilde{P}) f_{\mu \nu}-\tilde{P}_{\mu \nu} \tilde{P}_{\mu \nu}\right]+\mathcal{O}\left(\frac{\tilde{P}^{3}}{\tilde{\mu}^{6}}\right)
$$

where $\tilde{P}_{\mu \nu} \equiv P_{\mu \nu}(f)$ is the Schouten tensor for $f_{\mu \nu}$. Indices are now contracted with $f_{\mu \nu}$ and,

$$
\tilde{\mu}=\frac{m^{4}}{\alpha^{2} m_{g}^{2}}, \quad \tilde{s}_{n}=\sum_{k=n}^{3}\left(\begin{array}{l}
3-n \\
k-n
\end{array}\right) \tilde{a}^{k} \beta_{4-k},
$$

with $\tilde{a}$ being a solution to the polynomial equation $\tilde{s}_{0}=0$.

We can use the expression (A.5) for $f_{\mu \nu}$ to eliminate it from its own equation. For instance, inserting (A.5) into the Einstein tensor for $f_{\mu \nu}$ results in,

$$
\begin{aligned}
\tilde{\mathcal{G}}_{\mu \nu}(f)=\mathcal{G}_{\mu \nu}(g)-\frac{1}{s_{1} \mu^{2}}\left(\nabla^{2} P_{\mu \nu}\right. & +\nabla_{\mu} \nabla_{\nu} P-\nabla^{\rho} \nabla_{\mu} P_{\rho \nu}-\nabla^{\rho} \nabla_{\nu} P_{\rho \mu} \\
& \left.+3 P P_{\mu \nu}-g_{\mu \nu}\left[P^{\alpha \beta} P_{\alpha \beta}+\frac{1}{2} P^{2}\right]\right)+\mathcal{O}\left(\frac{P^{3}}{\mu^{4}}\right) .
\end{aligned}
$$

The contributions from the interaction potential read as,

$$
\begin{aligned}
\frac{\mu^{2}}{\alpha^{2}} \tilde{V}_{\mu \nu} & =\frac{\mu^{2} \Omega}{a^{2} \alpha^{2}} g_{\mu \nu}+\frac{1}{a^{2} \alpha^{2}} \mathcal{G}_{\mu \nu}+\frac{2 \Omega}{a^{2} \alpha^{2} s_{1}} P_{\mu \nu} \\
& +\frac{1}{a^{2} \alpha^{2} s_{1}^{3} \mu^{2}}\left[x_{1} P_{\mu}^{\rho} P_{\rho \nu}+x_{2} P P_{\mu \nu}+\frac{1}{6} g_{\mu \nu}\left(x_{3} P^{\alpha \beta} P_{\alpha \beta}-x_{2} P^{2}\right)\right]+\mathcal{O}\left(\frac{P^{3}}{\mu^{4}}\right),
\end{aligned}
$$

in which the coefficients $x_{n}$ are given by,

$$
x_{1}=2 s_{1}^{2}+\Omega\left(s_{1}+2 s_{2}\right), \quad x_{2}=-3 s_{1}^{2}-2 s_{2} \Omega, \quad x_{3}=3 s_{1}^{2}-2 s_{2} \Omega,
$$

and we have defined,

$$
\Omega=a \beta_{1}+3 a^{2} \beta_{2}+3 a^{3} \beta_{3}+a^{4} \beta_{4} .
$$


Combining the above results, the entire $f_{\mu \nu}$ equation of motion becomes the following higher derivative equation for $g_{\mu \nu}$,

$$
\begin{aligned}
& \frac{\Omega}{a^{2} \alpha^{2}} g_{\mu \nu}+\frac{1}{\mu^{2}}\left[1+\frac{1}{a^{2} \alpha^{2}}\right] \mathcal{G}_{\mu \nu}+\frac{2 \Omega}{a^{2} \alpha^{2} s_{1} \mu^{2}} P_{\mu \nu}+\frac{1}{\mu^{4} s_{1}} B_{\mu \nu} \\
& +\frac{\Omega}{a^{2} \alpha^{2} s_{1}^{3} \mu^{4}}\left[\left(s_{1}+2 s_{2}\right) P_{\mu}{ }^{\rho} P_{\rho \nu}-2 s_{2} P P_{\mu \nu}-\frac{s_{2}}{3} g_{\mu \nu}\left(P_{\rho \sigma} P^{\rho \sigma}-P^{2}\right)\right] \\
& -\frac{1}{s_{1} \mu^{4}}\left(1+\frac{1}{\alpha^{2} a^{2}}\right)\left[3 P P_{\mu \nu}-2 P_{\mu}{ }^{\rho} P_{\rho \nu}-\frac{1}{2} g_{\mu \nu}\left(P^{2}-P^{\alpha \beta} P_{\alpha \beta}\right)\right]+\mathcal{O}\left(\frac{P^{3}}{\mu^{6}}\right)=0 .
\end{aligned}
$$

Here, some of the four-derivative terms have been collected into the Bach tensor [1],

$$
B_{\mu \nu}=-\nabla^{2} P_{\mu \nu}-\nabla_{\mu} \nabla_{\nu} P_{\rho}^{\rho}+\nabla_{\rho} \nabla_{\mu} P_{\nu}^{\rho}+\nabla_{\rho} \nabla_{\nu} P_{\mu}^{\rho}-2 P_{\mu}^{\rho} P_{\rho \nu}+\frac{1}{2} g_{\mu \nu} P^{\rho \sigma} P_{\rho \sigma}
$$

which is invariant under local Weyl transformations of the metric.

\section{B Four-derivative terms in gauge transformations}

Here we outline the computation of the four-derivative terms generated by lower orders in the symmetry transformation.

\section{B.1 Higher-curvature equations}

We start by presenting the explicit expressions for the higher-curvature expansions of the equations of motion in the PM bimetric model up to 10th order in derivatives. The equations read,

$$
\begin{array}{ll}
f_{\mu \nu}^{\prime}=-g_{\mu \nu}^{\prime}+4 \rho P_{\mu \nu}+4 \sum_{n=2}^{\infty} \rho^{n} \gamma_{\mu \nu}^{(2 n)}\left[g^{\prime}\right], & \\
g_{\mu \nu}^{\prime}=-f_{\mu \nu}^{\prime}+4 \rho \tilde{P}_{\mu \nu}+4 \sum_{n=2}^{\infty} \rho^{n} \gamma_{\mu \nu}^{(2 n)}\left[f^{\prime}\right], & \rho \equiv \frac{\alpha}{4 \beta_{2} \mu^{2}} .
\end{array}
$$

Here, the higher-derivative functions $\gamma_{\mu \nu}^{(2 n)}$ for $n \leq 5$ are of the form, ${ }^{17}$

$$
\begin{aligned}
& \gamma_{\mu \nu}^{(4)}[g]=-2 P_{\mu \nu}^{2}+e_{1} P_{\mu \nu}-\frac{1}{3} e_{2} g_{\mu \nu} \\
& \gamma_{\mu \nu}^{(6)}[g]=4 P_{\mu \nu}^{3}-5 e_{1} P_{\mu \nu}^{2}+\left(e_{1}^{2}+2 e_{2}\right) P_{\mu \nu}-\left(e_{3}+\frac{1}{3} e_{1} e_{2}\right) g_{\mu \nu} \\
& \gamma_{\mu \nu}^{(8)}[g]=8 e_{1} P_{\mu \nu}^{3}-\left(9 e_{1}^{2}-\frac{8}{3} e_{2}\right) P_{\mu \nu}^{2}+\left(e_{1}^{3}+\frac{14}{3} e_{1} e_{2}-4 e_{3}\right) P_{\mu \nu} \\
&-\left(\frac{1}{3} e_{1}^{2} e_{2}-\frac{1}{9} e_{2}^{2}+\frac{8}{3} e_{1} e_{3}-\frac{20}{3} e_{4}\right) g_{\mu \nu}, \\
& \begin{aligned}
\gamma_{\mu \nu}^{(10)}[g]=\left(13 e_{1}^{2}-\frac{8}{3} e_{2}\right) P_{\mu \nu}^{3}-\left(14 e_{1}^{3}-7 e_{1} e_{2}-6 e_{3}\right) P_{\mu \nu}^{2} \\
+\left(e_{1}^{4}+8 e_{1}^{2} e_{2}+\frac{2}{3} e_{2}^{2}-13 e_{1} e_{3}-8 e_{4}\right) P_{\mu \nu} \\
-\left(\frac{1}{3} e_{1}^{3} e_{2}-\frac{1}{3} e_{1} e_{2}^{2}+5 e_{1}^{2} e_{3}+\frac{1}{3} e_{2} e_{3}-22 e_{1} e_{4}\right) g_{\mu \nu} .
\end{aligned}
\end{aligned}
$$

\footnotetext{
${ }^{17}$ We have made use of the Cayley-Hamilton theorem (A.4) to reduce the tensor powers in $\gamma_{\mu \nu}^{(8)}$ and $\gamma_{\mu \nu}^{(10)}$.
} 
In this expression all of the $e_{n}$ are elementary symmetric polynomials of the Schouten tensor $P_{\nu}^{\mu}=g^{\mu \rho} P_{\rho \nu}(g)$.

For future reference we also present the following linear variations of the four-derivative terms,

$$
\begin{aligned}
\delta \gamma_{\mu \nu}^{(4)}= & {\left[2 P_{\mu}{ }^{\rho} P_{\nu}^{\sigma}-P_{\mu \nu} P^{\rho \sigma}-\frac{1}{3} e_{2}(P) \delta_{\mu}^{\rho} \delta_{\nu}^{\sigma}+\frac{1}{3} g_{\mu \nu} P P^{\rho \sigma}-\frac{1}{3} g_{\mu \nu} P_{\lambda}^{\rho} P^{\lambda \sigma}\right] \delta g_{\rho \sigma} } \\
& +\left[P_{\mu \nu} g^{\rho \sigma}+P \delta_{\mu}^{\rho} \delta_{\nu}^{\sigma}-2 P_{\mu}{ }^{\rho} \delta_{\nu}^{\sigma}-2 P_{\nu}{ }^{\rho} \delta_{\mu}^{\sigma}-\frac{1}{3} g_{\mu \nu} P g^{\rho \sigma}+\frac{1}{3} g_{\mu \nu} P^{\rho \sigma}\right] \delta P_{\rho \sigma}
\end{aligned}
$$

where the linearised Schouten tensor reads as,

$$
\begin{aligned}
\delta P_{\mu \nu}= & P_{\mu \nu}[g+\delta g]-P_{\mu \nu}[g] \\
= & -\frac{1}{2}\left[\nabla_{\mu} \nabla_{\nu} \delta g-\nabla^{\rho} \nabla_{\mu} \delta g_{\nu \rho}-\nabla^{\rho} \nabla_{\nu} \delta g_{\mu \rho}+\nabla^{2} \delta g_{\mu \nu}\right]+\frac{1}{6} g_{\mu \nu}\left[\nabla^{2} \delta g-\nabla^{\rho} \nabla^{\sigma} \delta g_{\sigma \rho}\right] \\
& +\frac{1}{6} g_{\mu \nu} \delta g^{\rho \sigma} P_{\rho \sigma}-\frac{1}{2} P_{\rho}^{\rho}\left[\delta g_{\mu \nu}-\frac{1}{6} g_{\mu \nu} \delta g\right]
\end{aligned}
$$

and we have,

$$
\delta e_{n}(P)=\sum_{k=1}^{n}(-1)^{k} e_{n-k}(P)\left[\left[P^{k}\right]^{\rho \sigma} \delta g_{\rho \sigma}-\left[P^{k-1}\right]^{\rho \sigma} \delta P_{\rho \sigma}\right]
$$

These will be useful for computing the contribution to the four-derivative terms in the gauge transformations.

\section{B.2 Vanishing of the four-derivative contributions}

Consider arbitrary variations expanded in powers of derivatives up to $4^{\text {th }}$ order,

$$
\begin{aligned}
& \Delta g_{\mu \nu}^{\prime}=\Delta_{(0)} g_{\mu \nu}^{\prime}+\Delta_{(2)} g_{\mu \nu}^{\prime}+\Delta_{(4)} g_{\mu \nu}^{\prime}+\ldots \\
& \Delta f_{\mu \nu}^{\prime}=\Delta_{(0)} f_{\mu \nu}^{\prime}+\Delta_{(2)} f_{\mu \nu}^{\prime}+\Delta_{(4)} f_{\mu \nu}^{\prime}+\ldots
\end{aligned}
$$

The terms with zero and two derivatives were determined earlier,

$$
\begin{array}{ll}
\Delta_{(0)} g_{\mu \nu}^{\prime}=\phi g_{\mu \nu}^{\prime}, & \Delta_{(2)} g_{\mu \nu}^{\prime}=-2 \rho \phi P_{\mu \nu}-2 \rho \nabla_{\mu} \partial_{\nu} \phi, \\
\Delta_{(0)} f_{\mu \nu}^{\prime}=-\phi g_{\mu \nu}^{\prime}, & \Delta_{(2)} f_{\mu \nu}^{\prime}=2 \rho \phi P_{\mu \nu}-2 \rho \nabla_{\mu} \partial_{\nu} \phi .
\end{array}
$$

Using (B.1b) to replace $g_{\mu \nu}^{\prime}=-f_{\mu \nu}^{\prime}+4 \rho \tilde{P}_{\mu \nu}+\ldots$, the transformations of $f_{\mu \nu}^{\prime}$ can also be expressed in terms of $f_{\mu \nu}^{\prime}$,

$$
\Delta_{(0)} f_{\mu \nu}^{\prime}=\phi f_{\mu \nu}^{\prime}, \quad \Delta_{(2)} f_{\mu \nu}^{\prime}=-2 \rho \phi \tilde{P}_{\mu \nu}-2 \rho \tilde{\nabla}_{\mu} \partial_{\nu} \phi
$$


Demanding the invariance of (B.1a) at the four-derivative level and using the results from appendix B.1, we then obtain the following relation among the transformations,

$$
\begin{aligned}
\Delta_{(0)} f_{\mu \nu}^{\prime}+ & \Delta_{(2)} f_{\mu \nu}^{\prime}+\Delta_{(4)} f_{\mu \nu}^{\prime} \\
= & -\phi g_{\mu \nu}^{\prime}-\Delta_{(2)} g_{\mu \nu}^{\prime}-4 \rho \nabla_{\mu} \partial_{\nu} \phi-\Delta_{(4)} g_{\mu \nu}^{\prime}-4 \rho^{2} \gamma_{\mu \nu}^{(4)}\left[g^{\prime}\right] \phi \\
& -2 \rho\left[\nabla_{\mu} \nabla_{\nu} \Delta_{(2)} g_{\rho}^{\prime \rho}-\nabla^{\rho} \nabla_{\mu} \delta \Delta_{(2)} g_{\nu \rho}^{\prime}-\nabla^{\rho} \nabla_{\nu} \delta \Delta_{(2)} g_{\mu \rho}^{\prime}+\nabla^{2} \Delta_{(2)} g_{\mu \nu}^{\prime}\right] \\
& +\frac{2}{3} \rho g_{\mu \nu}^{\prime}\left[\nabla^{2} \Delta_{(2)} g_{\rho}^{\prime \rho}-\nabla^{\rho} \nabla^{\sigma} \Delta_{(2)} g_{\rho \sigma}^{\prime}\right]+\frac{2}{3} \rho g_{\mu \nu}^{\prime} P^{\rho \sigma} \Delta_{(2)} g_{\rho \sigma}^{\prime} \\
& -2 \rho P\left[\Delta_{(2)} g_{\mu \nu}^{\prime}-\frac{1}{6} g_{\mu \nu}^{\prime} \Delta_{(2)} g_{\rho}^{\prime \rho}\right]-4 \rho^{2}\left[P_{\mu \nu} \nabla^{2} \phi+P \nabla_{\mu} \nabla_{\nu} \phi\right. \\
& \left.-2 P_{\mu}^{\rho} \nabla_{\rho} \nabla_{\nu} \phi-2 P_{\nu}^{\rho} \nabla_{\rho} \nabla_{\mu} \phi-\frac{1}{3} g_{\mu \nu}^{\prime} P \nabla^{2} \phi+\frac{1}{3} g_{\mu \nu}^{\prime} P^{\rho \sigma} \nabla_{\rho} \nabla_{\sigma} \phi\right],
\end{aligned}
$$

where all quantities on the right hand side are given in terms of $g_{\mu \nu}^{\prime}$. We now use (B.1b) to express the right hand side of (B.12) as a functional of $f_{\mu \nu}^{\prime}$,

$$
\begin{aligned}
\Delta_{(0)} f_{\mu \nu}^{\prime}+ & \Delta_{(2)} f_{\mu \nu}^{\prime}+\Delta_{(4)} f_{\mu \nu}^{\prime} \\
= & \phi f_{\mu \nu}^{\prime}-4 \rho \phi \tilde{P}_{\mu \nu}-4 \rho \tilde{\nabla}_{\mu} \partial_{\nu} \phi+4 \rho C_{\mu \nu}{ }^{\rho} \tilde{\nabla}_{\rho} \phi-\left.\Delta_{(2)} g_{\mu \nu}^{\prime}\right|_{g^{\prime}=-f^{\prime}+4 \rho \tilde{P}}+\left.\Delta_{(4)} g_{\mu \nu}^{\prime}\right|_{g^{\prime}=-f^{\prime}} \\
& +2 \rho\left[\tilde{\nabla}_{\mu} \tilde{\nabla}_{\nu} \Delta_{(2)} g_{\rho}^{\rho \rho}-\tilde{\nabla}^{\rho} \tilde{\nabla}_{\mu} \Delta_{(2)} g_{\nu \rho}^{\prime}-\tilde{\nabla}^{\rho} \tilde{\nabla}_{\nu} \Delta_{(2)} g_{\mu \rho}^{\prime}+\tilde{\nabla}^{2} \Delta_{(2)} g_{\mu \nu}^{\prime}\right] \\
& -\frac{2 \rho}{3} g_{\mu \nu}^{\prime}\left[\tilde{\nabla}^{2} \Delta_{(2)} g_{\rho}^{\prime \rho}-\tilde{\nabla}^{\rho} \tilde{\nabla}^{\sigma} \Delta_{(2)} \tilde{g}_{\rho \sigma}\right]-\frac{2 \rho}{3} g_{\mu \nu}^{\prime} \tilde{P}^{\rho \sigma} \Delta_{(2)} g_{\rho \sigma}^{\prime} \\
& +2 \rho \tilde{P}\left[\Delta_{(2)} g_{\mu \nu}^{\prime}-\frac{1}{6} g_{\mu \nu}^{\prime} \Delta_{(2)} g_{\rho}^{\prime \rho}\right]+4 \rho^{2}\left[\tilde{P}_{\mu \nu} \tilde{\nabla}^{2} \phi+\tilde{P} \tilde{\nabla}_{\mu} \partial_{\nu} \phi\right. \\
& \left.-2 \tilde{P}_{\mu \rho} \tilde{\nabla}^{\rho} \partial_{\nu} \phi-2 \tilde{P}_{\nu \rho} \tilde{\nabla}^{\rho} \partial_{\mu} \phi-\frac{1}{3} g_{\mu \nu}^{\prime} \tilde{P} \tilde{\nabla}^{2} \phi+\frac{1}{3} g_{\mu \nu}^{\prime} \tilde{P}^{\rho \sigma} \tilde{\nabla}_{\rho} \partial_{\sigma} \phi\right],
\end{aligned}
$$

where now, on the right-hand side, all quantities (curvatures, derivatives, index raising, etc.) are understood to be defined with respect to $f_{\mu \nu}^{\prime}$ and in the transformations $g_{\mu \nu}^{\prime}$ is a function of $f_{\mu \nu}^{\prime}$,

$$
\Delta_{(2)} g_{\mu \nu}^{\prime}=\left.\Delta_{(2)} g_{\mu \nu}^{\prime}\right|_{g^{\prime}=-f^{\prime}+4 \rho \tilde{P}}, \quad \Delta_{(4)} g_{\mu \nu}^{\prime}=\left.\Delta_{(4)} g_{\mu \nu}^{\prime}\right|_{g^{\prime}=-f^{\prime}} .
$$

Furthermore, we have introduced

$$
C_{\mu \nu}{ }^{\rho}=-2 \rho\left[\tilde{\nabla}_{\mu} \tilde{P}_{\nu}^{\rho}+\tilde{\nabla}_{\nu} \tilde{P}_{\mu}^{\rho}-\tilde{\nabla}^{\rho} \tilde{P}_{\mu \nu}\right] .
$$

Next, we try to impose the interchange symmetry of the transformations. According to our discussion in section 4.3 , this is only possible if the contributions to the transformations that are generated from lower orders vanish. Indeed, if we demand,

$$
\Delta_{(4)} f_{\mu \nu}^{\prime}=\left.\Delta_{(4)} g_{\mu \nu}^{\prime}\right|_{g^{\prime} \leftrightarrow f^{\prime}},
$$

after a lengthy but straightforward calculation we find that this condition becomes,

$$
\begin{aligned}
\Delta_{(4)} g_{\mu \nu}^{\prime}=\Delta_{(4)} g_{\mu \nu}^{\prime} & +4 \rho^{2}\left[\nabla_{\rho} \nabla_{\mu} \nabla_{\nu} \partial^{\rho} \phi+\nabla^{\rho} \nabla_{\nu} \nabla_{\rho} \partial_{\mu} \phi-\nabla_{\mu} \nabla_{\nu} \nabla^{2} \phi-\nabla^{2} \nabla_{\mu} \partial_{\nu} \phi\right] \\
& -4 \rho^{2}\left[P \nabla_{\mu} \partial_{\nu} \phi+\nabla_{\rho} P_{\mu \nu} \partial^{\rho} \phi+P_{\mu \rho} \nabla^{\rho} \partial_{\nu} \phi+P_{\nu \rho} \nabla^{\rho} \partial_{\mu} \phi\right] \\
& -\frac{4 \rho}{3} g_{\mu \nu}^{\prime}\left[\nabla_{\rho} \nabla_{\sigma} \nabla^{\rho} \partial^{\sigma} \phi-\nabla^{2} \nabla^{2} \phi-P^{\rho \sigma} \nabla_{\rho} \partial_{\sigma} \phi-\frac{1}{2} P \nabla^{2} \phi\right]
\end{aligned}
$$


Clearly, this leaves $\Delta_{(4)} g_{\mu \nu}^{\prime}$ undetermined and instead provides a consistency check on the possible existence of a symmetry to this order in derivatives. Obviously, the condition is satisfied for constant gauge parameter, but showing that the extra terms vanish for a general function $\phi$ requires a bit more work.

Some identities that are useful for our purposes at this point are, ${ }^{18}$

$$
\begin{aligned}
\nabla_{\rho} \nabla_{\mu} \nabla_{\nu} \nabla^{\rho} \phi=\nabla_{\mu} \nabla_{\nu} \nabla^{2} \phi & +R_{\rho \mu \nu}{ }^{\sigma} \nabla_{\sigma} \nabla^{\rho} \phi+P \nabla_{\mu} \nabla_{\nu} \phi+\frac{1}{2} \nabla_{\mu} P \nabla_{\nu} \phi \\
& +P_{\mu}{ }^{\rho} \nabla_{\nu} \nabla_{\rho} \phi+P_{\nu}{ }^{\rho} \nabla_{\mu} \nabla_{\rho} \phi+\nabla_{\mu} P_{\nu \rho} \nabla^{\rho} \phi, \\
\nabla^{\rho} \nabla_{\nu} \nabla_{\rho} \nabla_{\mu} \phi=\nabla^{2} \nabla_{\mu} \nabla_{\nu} \phi-R_{\rho \mu \nu}{ }^{\sigma} \nabla_{\sigma} \nabla^{\rho} \phi+\nabla^{\rho} R_{\nu \rho \mu}{ }^{\sigma} \nabla_{\sigma} \phi & \\
\nabla_{\rho} \nabla_{\sigma} \nabla^{\rho} \nabla^{\sigma} \phi= & \nabla^{2} \nabla^{2} \phi+P^{\rho \sigma} \nabla_{\rho} \nabla_{\sigma} \phi+\frac{1}{2} P \nabla^{2} \phi+\frac{3}{2} \nabla_{\rho} P \nabla^{\rho} \phi .
\end{aligned}
$$

Using these identities, we can write the condition (B.17) as,

$$
\nabla_{\rho} R_{\mu \sigma \nu}^{\rho} \nabla^{\sigma} \phi+\nabla_{\mu} P_{\nu \sigma} \nabla^{\sigma} \phi-\nabla_{\sigma} P_{\mu \nu} \nabla^{\sigma} \phi+\frac{1}{2} \nabla_{\mu} P \nabla_{\nu} \phi-\frac{1}{2} g_{\mu \nu}^{\prime} \nabla_{\sigma} P \nabla^{\sigma} \phi=0 .
$$

Now, in order to show that this is always true, consider the (contracted) second Bianchi identity,

$$
\nabla_{\rho} R_{\mu \sigma \nu}^{\rho}+\nabla_{\mu} R_{\nu \sigma}-\nabla_{\sigma} R_{\mu \nu}=0
$$

or equivalently, using $R_{\mu \nu}=P_{\mu \nu}+\frac{1}{2} g_{\mu \nu} P$,

$$
\nabla_{\rho} R_{\mu \sigma \nu}^{\rho}+\nabla_{\mu} P_{\nu \sigma}-\nabla_{\sigma} P_{\mu \nu}+\frac{1}{2} g_{\nu \sigma} \nabla_{\mu} P-\frac{1}{2} g_{\mu \nu} \nabla_{\sigma} P=0 .
$$

Using this, it is obvious that (B.21) and hence (B.17) are identically satisfied for any function $\phi$. This proves that there are no four-derivative terms generated from lower orders in the gauge transformations and hence the condition for the existence of an invariance is satisfied at the first nontrivial order.

\section{References}

[1] R. Bach, Mathematische Zeitschrift, 9 (1921) 110.

[2] K. S. Stelle, Phys. Rev. D 16 (1977) 953.

[3] M. Kaku, P. K. Townsend and P. van Nieuwenhuizen, Phys. Lett. B 69 (1977) 304.

[4] S. Ferrara and B. Zumino, Nucl. Phys. B 134 (1978)

[5] E. S. Fradkin and A. A. Tseytlin, Nucl. Phys. B 201 (1982) 469.

[6] R. J. Riegert, Phys. Lett. A 105 (1984) 110.

[7] J. Maldacena, arXiv:1105.5632 [hep-th].

\footnotetext{
${ }^{18}$ Note that our curvature conventions are $\left[\nabla_{\mu}, \nabla_{\nu}\right] \omega_{\rho}=R_{\mu \nu \rho}{ }^{\sigma} \omega_{\sigma}$.
} 
[8] A. A. Tseytlin, Phys. Lett. B 363 (1995) 223 [hep-th/9509050].

[9] T. Biswas, A. Mazumdar and W. Siegel, JCAP 0603 (2006) 009 [hep-th/0508194].

[10] T. Biswas, E. Gerwick, T. Koivisto and A. Mazumdar, Phys. Rev. Lett. 108 (2012) 031101 [arXiv:1110.5249 [gr-qc]].

[11] S. Deser and R. I. Nepomechie, Annals Phys. 154 (1984) 396.

[12] S. Deser and A. Waldron, Phys. Rev. Lett. 87 (2001) 031601 [hep-th/0102166].

[13] S. Deser and A. Waldron, Nucl. Phys. B 607 (2001) 577 [hep-th/0103198].

[14] Y. M. Zinoviev, Nucl. Phys. B 770 (2007) 83 [hep-th/0609170].

[15] Y. M. Zinoviev, Nucl. Phys. B 872 (2013) 21 [arXiv:1302.1983 [hep-th]].

[16] E. Joung, L. Lopez and M. Taronna, JHEP 1207 (2012) 041 [arXiv:1203.6578 [hep-th]].

[17] S. F. Hassan and R. A. Rosen, JHEP 1202 (2012) 126 [arXiv:1109.3515 [hep-th]].

[18] S. F. Hassan, A. Schmidt-May and M. von Strauss, Phys. Lett. B 726, (2013) 834 [arXiv:1208.1797 [hep-th]].

[19] S. F. Hassan, A. Schmidt-May and M. von Strauss, Class. Quant. Grav. 30 (2013) 184010 [arXiv:1212.4525 [hep-th]].

[20] S. F. Hassan, A. Schmidt-May and M. von Strauss, Universe 1 (2015) 2, 92 doi:10.3390/universe1020092 [arXiv:1303.6940 [hep-th]].

[21] C. de Rham, G. Gabadadze and A. J. Tolley, Phys. Rev. Lett. 106 (2011) 231101 [arXiv:1011.1232 [hep-th]].

[22] S. F. Hassan and R. A. Rosen, Phys. Rev. Lett. 108 (2012) 041101 [arXiv:1106.3344 [hep-th]].

[23] S. F. Hassan and R. A. Rosen, JHEP 1107 (2011) 009. [arXiv:1103.6055 [hep-th]].

[24] S. F. Hassan, R. A. Rosen and A. Schmidt-May, JHEP 1202 (2012) 026 [arXiv:1109.3230 [hep-th]].

[25] S. F. Hassan, A. Schmidt-May and M. von Strauss, Phys. Lett. B 715 (2012) 335 [arXiv:1203.5283 [hep-th]].

[26] C. de Rham and S. Renaux-Petel, JCAP 1301 (2013) 035 doi:10.1088/1475-7516/2013/01/035 [arXiv:1206.3482 [hep-th]].

[27] S. Deser, M. Sandora and A. Waldron, Phys. Rev. D 87 (2013) 101501 [arXiv:1301.5621 [hep-th]].

[28] C. de Rham, K. Hinterbichler, R. A. Rosen and A. J. Tolley, Phys. Rev. D 88 (2013) 024003 [arXiv:1302.0025 [hep-th]].

[29] S. Deser, M. Sandora and A. Waldron, Phys. Rev. D 88 (2013) 081501 [arXiv:1306.0647 [hep-th]].

[30] D. Comelli, F. Nesti and L. Pilo, JCAP 1411 (2014) 11, 018 [arXiv:1407.4991 [hep-th]].

[31] S. F. Hassan, A. Schmidt-May and M. von Strauss, arXiv:1407.2772 [hep-th].

[32] E. Joung, W. Li and M. Taronna, Phys. Rev. Lett. 113 (2014) 091101 [arXiv:1406.2335 [hep-th]]. 
[33] S. Alexandrov and C. Deffayet, JCAP 1503 (2015) 03, 043 [arXiv:1410.2897 [hep-th]].

[34] K. Hinterbichler, Phys. Rev. D 91 (2015) 2, 026008 [arXiv:1409.3565 [hep-th]].

[35] S. Garcia-Saenz and R. A. Rosen, JHEP 1505 (2015) 042 [arXiv:1410.8734 [hep-th]].

[36] C. de Rham and G. Gabadadze, Phys. Rev. D 82 (2010) 044020 [arXiv:1007.0443 [hep-th]].

[37] S. F. Hassan and R. A. Rosen, JHEP 1204 (2012) 123 [arXiv:1111.2070 [hep-th]].

[38] D. G. Boulware and S. Deser, Phys. Lett. B 40 (1972) 227.

[39] D. G. Boulware and S. Deser, Phys. Rev. D 6 (1972) 3368.

[40] S. F. Hassan, A. Schmidt-May and M. von Strauss, JHEP 1305 (2013) 086 [arXiv:1208.1515 [hep-th]].

[41] Y. Akrami, S. F. Hassan, F. Knnig, A. Schmidt-May and A. R. Solomon, Phys. Lett. B 748 (2015) 37 doi:10.1016/j.physletb.2015.06.062 [arXiv:1503.07521 [gr-qc]].

[42] A. Higuchi, Nucl. Phys. B 282 (1987) 397.

[43] S. Deser, E. Joung and A. Waldron, J. Phys. A 46 (2013) 214019 doi:10.1088/1751-8113/46/21/214019 [arXiv:1208.1307 [hep-th]].

[44] M. von Strauss, A. Schmidt-May, J. Enander, E. Mortsell and S. F. Hassan, JCAP 1203 (2012) 042 [arXiv:1111.1655 [gr-qc]].

[45] G. 't Hooft, Found. Phys. 41 (2011) 1829 [arXiv:1104.4543 [gr-qc]].

[46] G. T. Hooft, arXiv:1410.6675 [gr-qc].

[47] V. Baccetti, P. Martin-Moruno and M. Visser, Class. Quant. Grav. 30 (2013) 015004 [arXiv:1205.2158 [gr-qc]]. 